\title{
Diatom (Bacillariophyceae) flora of early Holocene freshwater sediments from Skalafjord, Faeroe Islands
}

\author{
EWA WITON ${ }^{1} \&$ ANDRZEJ WITKOWSKI ${ }^{2}$ \\ ${ }^{1}$ Department of Earth Sciences - Marine Geology, University of Göteborg, Box 460, SE-405 30 Göteborg, Sweden (e-mail: ewa@gvc.gu.se) \\ ${ }^{2}$ Department of Paleoceanology, University of Szczecin, Waska 13, PL-71 415 Szczecin, Poland (e-mail: witkowsk@univ.szczecin.pl).
}

\begin{abstract}
Relative abundance data of diatom (Bacillariophyceae) species were generated for sediment core SKPC-01B from the Skalafjord, Faeroe Islands. The record shows distinct temporal changes in species composition. In the lowermost $65 \mathrm{~cm}$ of the $230 \mathrm{~cm}$ long core a species-rich freshwater diatom assemblage was found. Most of the taxa observed in this section are typical of oligotrophic to dystrophic lakes in northern Europe (Scandinavia, Iceland and Spitsbergen). Above this interval the diatom flora is dominated by marine taxa. The change from a freshwater to a marine flora is inferred to be caused by rising sea-level that took place about 7700-6400 years BP. Drastic changes in the diatom species composition within the transitional core section show that environmental change in the Skalafjord took place in several pulses. The first stage included strong inflow (possibly catastrophic) of marine waters. As a possible trigger of this phenomenon the tsunami released by the Storegga Slide is proposed. Before the final flooding by marine waters, freshwater conditions were re-established within the Skalafjord. These results have important implications for the interpretation of the palaeogeographical development of the Eysturoy area. Hence, it is suggested that the Storegga Slide led to inflow of marine waters at a distinctly lower water level in the area of the Skalafjord than proposed in recent publications and that the inundation of the threshold in the fjord happened after the tsunami. J. Micropalaeontol. 22(2): 183-208, November 2003.
\end{abstract}

\section{INTRODUCTION}

Fossil diatom floras of freshwater and marine origin may be used for reconstructing environmental changes. Freshwater diatom floras from limnic sediments are useful for reconstructions of the climate changes that took place at high latitudes in the Northern Hemisphere since the last deglaciation. Recently, studies of lacustrine sediments from the land areas surrounding the North Sea and Norwegian Sea have been shown to contain a record of past catastrophic events that took place in the area. One such record is a tsunami caused by the Storegga Slide dated at c. $7500{ }^{14} \mathrm{C}$ years BP (Dawson \& Smith, 2000). Diatoms represent one of the best indicators of the impact of this tsunami on the sedimentary record. This phenomenon has been shown to occur in sediment cores from lakes from the Faeroe Islands (Grauert et al., 2001). Abrupt changes in diatom species composition were interpreted as indicators of this catastrophic event. However, the altitude of the sediment section analysed here and its significance for the ocean level at which the Storegga Slide took part is not in agreement with palaeogeographical interpretations given by Bennike et al. (1998) and Grauert et al. (2001).

Although the first publications on freshwater diatoms from the northern part of the North Atlantic are from the nineteenth and twentieth centuries, knowledge of the freshwater diatom flora of the Faeroe Islands is rather poor. Early publications (Lyngbye, 1819; Cleve, 1873, 1896, 1898, 1900; Lagerstedt, 1873; Cleve \& Grunow, 1880; Østrup, 1897; Brun, 1901) dealt with the high latitude North Atlantic in general and usually concerned both marine and freshwater floras. Later, Hustedt (1937), Krasske (1938) and Foged $(1964,1974)$ published results on their studies of the freshwater diatom flora from some North Atlantic islands (for example, the Faeroe Islands and Spitsbergen). Only Hustedt (1937) dealt with diatoms from Iceland, the Faeroe Islands and Spitsbergen. The first report focusing on freshwater diatoms from the Faeroe Islands was by Lyngbye (1819). The next study specifically dealing with the freshwater diatom flora from the Faeroe Islands was published by Østrup (1901). Somewhat later, Østrup (1903) published a report on marine diatoms from this area. Since then, no papers on freshwater diatoms from the Faeroes have been published, to the best of our knowledge.

Recently, an effort was directed towards studies of the marine diatom flora of the North Atlantic and the results were used for studies of climate change following the last deglaciation. The major objective of these studies was to decipher palaeoceanographical changes (Koc \& Schrader, 1990; Koc \& Jansen, 1992; Schrader et al., 1993a, b; Kohly, 1998; Wachnicka, 1999; Jozkow, 2000; Jiang et al., 2001; Witak et al., 2004).

Core SKPC-01B from the Skalafjord, Faeroe Islands has been analysed for diatoms. The Skalafjord penetrates into Eysturoy, the biggest of the Faeroe Islands (Fig. 1). Diatoms are well preserved and dominated by freshwater forms in the lowermost part and marine forms in the upper part. The focus is on the freshwater diatom flora from the lower part of the core. The sediments are of early Holocene age, and the flora is typical of high latitude nutrient-poor (oligotrophic to dystrophic) lakes (e.g. Cleve-Euler, 1951-1955; Lange-Bertalot \& Metzeltin, 1996). The position of the freshwater deposits within the section and the weak representation of marine elements suggest that deposition took place before the threshold in the Skalafjord was inundated.

\section{Geological setting}

The Faeroe Islands consist of a group of islands in the North Atlantic situated between $61^{\circ} 20^{\prime} \mathrm{N}$ and $62^{\circ} 24^{\prime} \mathrm{N}$ and between $6^{\circ} 15^{\prime} \mathrm{W}$ and $7^{\circ} 41^{\prime} \mathrm{W}$ (Fig. 1). Geologically, they belong to 


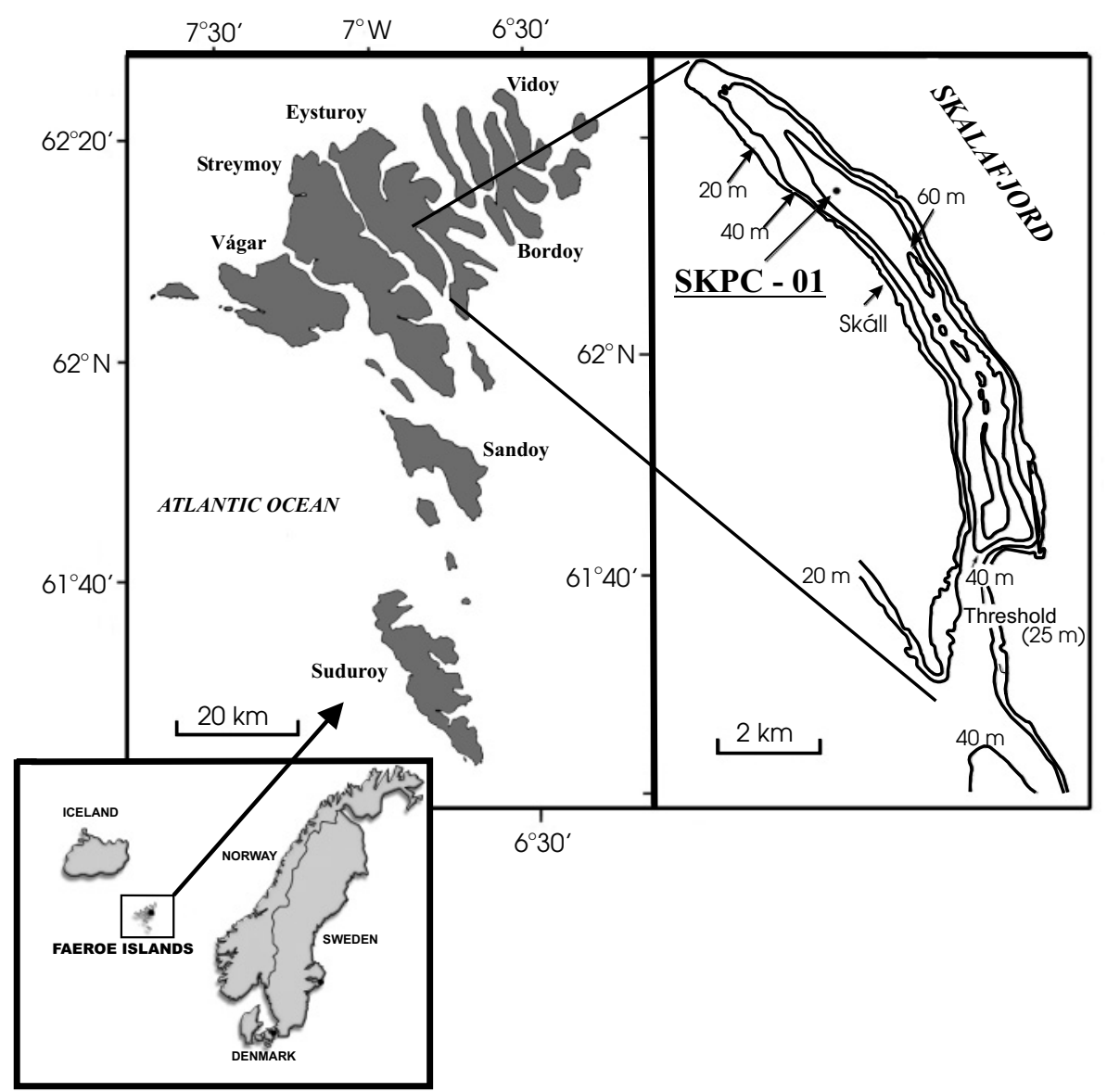

Fig. 1. Location of the core sampled in the Skalafjord, Faeroe Island.

the North Atlantic basalt province, and the whole area was influenced by Tertiary volcanism (Boldreel \& Andersen, 1995; van Weering et al., 1998). During glacial periods, part of the North Faeroe shelf was exposed subaerially and may have been glaciated (Jørgensen \& Rasmussen, 1986). Lakes and bogs are common throughout the islands, and the deposits within them provide Holocene palaeoenvironmental records (Grauert et al., 2001). Skalafjord is $13 \mathrm{~km}$ long with a greatest depth of around $70 \mathrm{~m}$. At the entrance of the fjord a sill with a water depth of $25 \mathrm{~m}$ is present. The fjord is surrounded by $500-600 \mathrm{~m}$ high mountains. Post-glacial sediments in the fjord were deposited in two separate basins and have a maximum thickness of about 20 m (Juul, 1992).

\section{MATERIAL AND METHODS}

Core SKPC-01B was one of nine sediment cores retrieved from the Faeroe Islands during the September-October $1995 \mathrm{R} / \mathrm{V}$ Skagerak cruise organized by Göteborg University in collaboration with the Geological Survey of Denmark and Greenland (Fig. 1). The coring site was situated in the central part of the Skalafjord $\left(62^{\circ} 10^{\prime} 70^{\prime \prime} \mathrm{N}\right.$ and $\left.6^{\circ} 47^{\prime} 87^{\prime \prime} \mathrm{W}\right)$ in a water depth of $50 \mathrm{~m}$.

Samples were prepared in the manner of Håkansson \& Ross (1984). Samples for diatom analyses were collected at $5 \mathrm{~cm}$ intervals. One gram of sediment was dried at $60^{\circ}$ for 24 hours. The sediment was treated with $10 \% \mathrm{HCl}$ to dissolve carbonates and then washed several times with distilled water. The siliceous material was gently boiled in concentrated $(37 \%) \mathrm{H}_{2} \mathrm{O}_{2}$ and washed several times with distilled water. The supernatant was decanted off after 20 hours. An aliquot of the shaken suspension was transferred by pipette to an $18 \times 18 \mathrm{~mm}$ square coverslip. The coverslips were left to dry at room temperature. After evaporation, the coverslips were placed onto labelled slides. Permanent diatom preparations were mounted with Naphrax $^{\mathbb{R}}$ (refractive index $=1.78$ ) and briefly heated to $200^{\circ} \mathrm{C}$. Diatom analyses were performed with a LEICA DMLB light microscope, using $\times 100 / 1.25$ planapochromatic oil-immersion objective. Scanning electron microscope analysis was performed by means of a Zeiss DSM 940 at $25 \mathrm{kV}$. In each sample more than 300 valves were counted. Diatoms were counted by the Schrader \& Gersonde (1978) method.

Diatom identifications were based on the works of Podzorski (1985), Krammer \& Lange-Bertalot (1986, 1991a, b, 1997, 2000), Sala et al. (1993), Lange-Bertalot \& Moser (1994), Lange-Bertalot \& Metzeltin (1996), Metzeltin \& Witkowski (1996), Witkowski et al. (1996), Metzeltin \& Lange-Bertalot (1998), Lange-Bertalot \& Genkal (1999), Reichardt (1999), Lange-Bertalot (2001), Krammer (1992, 1997, 2000, 2002) and Håkansson $(1990,2002)$ for freshwater taxa and Witkowski et al. (2000) for marine forms. Diatoms were divided into groups according to their ecological requirements after Denys (1992), Hoffman (1994) and Van Dam et al. (1994). 


\begin{tabular}{lcccr}
\hline $\begin{array}{l}\text { Depth } \\
(\mathrm{cm})\end{array}$ & Lab. no. & $\begin{array}{c}{ }^{14} \mathrm{C} \text { age } \\
(\mathrm{bp})\end{array}$ & $\begin{array}{c}\text { Reservoir-corrected } \\
(\mathrm{bp})\end{array}$ & $\begin{array}{c}{ }^{14} \mathrm{C} \text { age } \\
(\mathrm{bc})\end{array}$ \\
\hline 50 & AAR-6940 & $3380 \pm 55$ & $2980 \pm 55$ & $1370-1130$ \\
150 & AAR-6941 & $6235 \pm 60$ & $5835 \pm 60$ & $4780-4620$ \\
183 & AAR-6942 & $7465 \pm 55$ & $7065 \pm 55$ & $5990-5840$ \\
\hline
\end{tabular}

The calibrated ages in calendar years have been obtained from the calibration tables in Stuiver et al. (1998) by means of the 1998 version (4.0) of the Seattle CALIB program (Stuiver \& Reimer, 1993).

Table 1. Results of AMS ${ }^{14} \mathrm{C}$ datings for the piston core SKPC-01B retreived from the Skalafjord, Faeroe Islands

The chronology of core SKPC-01B is based on three radiocarbon AMS ${ }^{14} \mathrm{C}$ analyses (Table 1) from macrofossil shells. The dating was performed at the University of Aarhus, Denmark. Calibrated ages in calendar years were obtained from Stuiver et al. (1998) by means of the Seattle calibration program CALIB version 4.0 (Stuiver \& Reimer, 1993). Ages of certain levels in SKPC-01B were estimated through linear interpolation between the AMS ${ }^{14} \mathrm{C}$ dated levels assuming a constant sedimentation rate.

\section{DISTRIBUTION OF FRESHWATER DIATOMS}

\section{Abundance and concentration}

The core length studied is $230 \mathrm{~cm}$, and it is characterized by predominantly homogeneous olive-grey clayey mud (Fig. 2). More or less corroded shell fragments were observed along the whole profile. Their quantity distinctly increased at $120-130 \mathrm{~cm}$ depth. At $108-118 \mathrm{~cm}$ the sediment was distinctly laminated and somewhat darker.

Within the whole sediment profile the diatoms represent two completely different environments. At a depth of $230-165 \mathrm{~cm}$, taxa typical of limnic environments predominated (Fig. 3). Above $165 \mathrm{~cm}$ the flora is almost exclusively marine. The sediments representing these two different environments are connected by an apparently transitional section between 180 $165 \mathrm{~cm}$. In this part of the sediment profile a transition from limnic to marine conditions is recorded. First, in the section from $180-170 \mathrm{~cm}$, a strong peak in marine diatoms occurs followed by a dominance of freshwater taxa in the sediment interval from $170-165 \mathrm{~cm}$. These abrupt environmental changes took place during the period 7700-6400 years BP.

A total of 166 diatom taxa have been identified. In general, the preservation state was satisfactory but, at some levels, the valves were fragmented. Freshwater diatoms were represented by 121 taxa, brackish-water forms by 16 taxa and marine forms by 28 taxa. The freshwater flora was dominated by benthic species (126 species), while the planktonic flora consisted of 39 species.

In this paper the freshwater diatoms that occurred in the lowermost part of the core are described. Two diatom assemblage zones (DAZ) and several subzones are distinguished (Fig. 3). The first zone (DAZ-1) corresponds to the lower part of the core $(230-165 \mathrm{~cm})$. Two subzones were distinguished, DAZ-1a (depth interval 230-195 cm) and DAZ-1b (depth interval $195-165 \mathrm{~cm}$ ) (Fig. 4). The following criteria were applied to distinguish the diatom assemblage zones:

- changes in the ratio between marine and freshwater taxa;

- habitat characteristics, i.e. planktonic versus benthic forms;
- diatom concentration in number of valves per $1 \mathrm{~g}$ of sediment.

DAZ-1a. The age of the boundary between subzone DAZ-1a and DAZ-1b sediments was estimated to be about $7700{ }^{14} \mathrm{C}$ years BP. Diatom zone DAZ-1 is characterized by abundant freshwater taxa and less abundant marine ones (Fig. 3). Diatom valves are usually very well preserved.

In diatom subzone DAZ-1a the proportion of marine taxa was very low. Planktonic forms showed a distinct upward

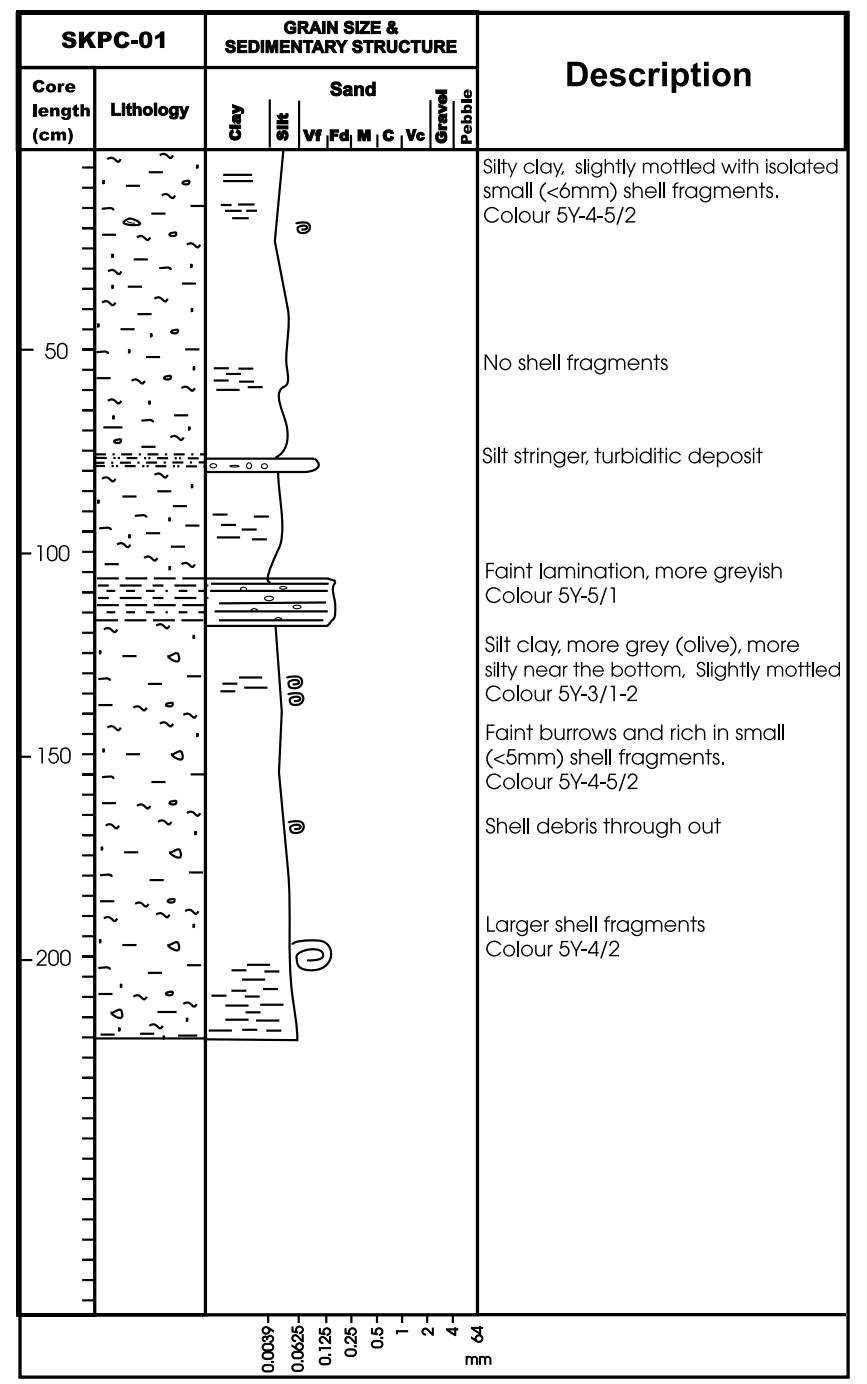

Fig. 2. Lithology of the core SKPC-01B. 


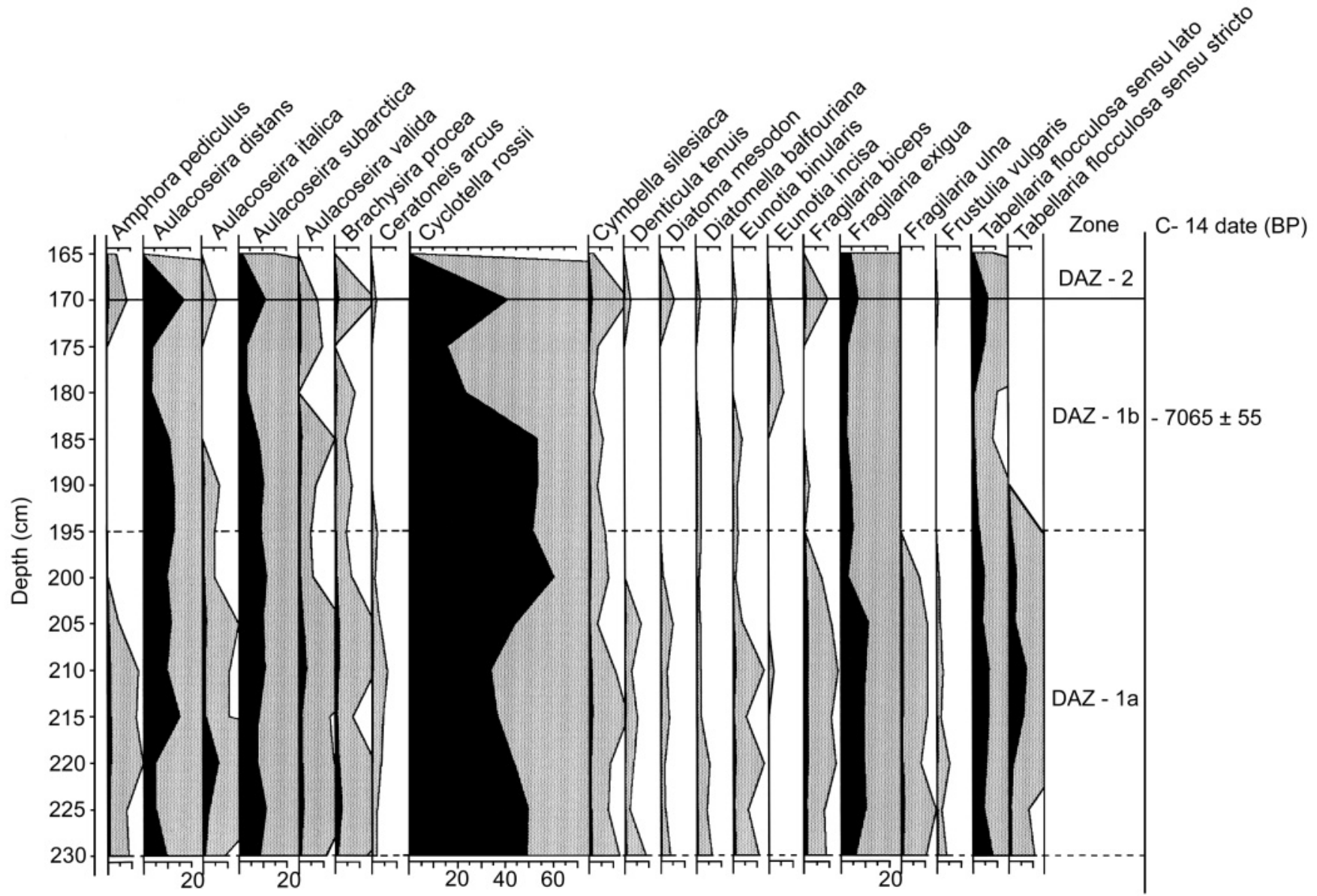

Fig. 3. Distribution and relative abundance the most common freshwater diatom taxa from core SKPC-01B from interval $165-230 \mathrm{~cm}$. Solid area stands for $\%$ contents of ecological groups. dotted areas express contents of ecological groups with very low abundance in $\%$.

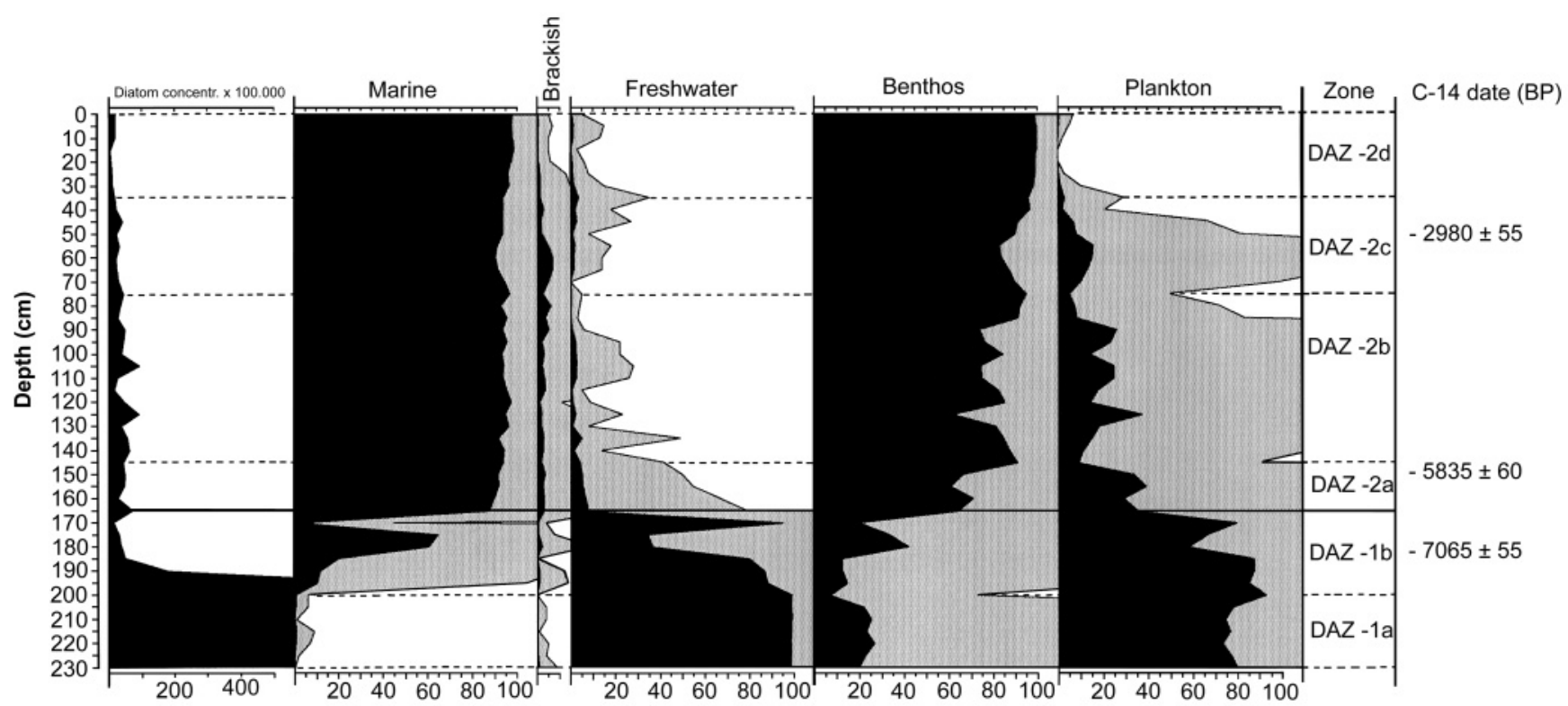

Fig. 4. Percentage diagram of diatom ecological groups core in SKPC-01B. Solid area stands for \% contents of particular taxa. dotted areas express contents of taxa with very low abundance in $\%$. 
increase within this subzone. The concentration of diatom valves fell from $435 \times 10^{6}$ to $3.5 \times 10^{6}$ per $1 \mathrm{~g}$ of sediment. The most abundant taxa include Cyclotella rossii Håkansson (up to 60\%), Aulacoseira distans (Ehrenberg) Simonsen (16\%), Fragilaria exigua Grunow (12\%), Aulacoseira subarctica (O. Müller) Haworth (12\%) and Tabellaria flocculosa (Roth) Kützing (7\%).

DAZ-1b. Diatom assemblage subzone DAZ-1b encompasses the sediment interval $195-165 \mathrm{~cm}$. The age of the transition from subzone DAZ-1b to DAZ-2 was estimated to be about 6400 years BP. The bottom of the zone is marked by a drastic decrease in diatom valve concentration to $c .50 \times 10^{5}$ valves $/ \mathrm{g}$ (Fig. 3). Freshwater taxa dominate, but in the interval 180 $170 \mathrm{~cm}$ a peak of marine taxa is recorded. Amongst the predominant species are: Paralia sulcata (up to 30\%), Thalassiosira nordenskioeldii (up to 17\%), Thalassiosira hyalina (up to $9 \%$ ) and Odontella aurita (up to 5\%). Among the freshwater forms the following taxa were the most abundant: C. rossii (up to $53 \%$ ), A. distans (up to $15 \%$ ), A. subarctica (up. to 10\%), Fragilaria ulna (up to $10 \%$ ) and T. flocculosa (up to 10\%). With respect to habitat, planktonic forms dominate with $50-80 \%$ (Fig. 4). Only in the section rich in marine forms is a peak in benthic forms (up to $40 \%$ ) seen.

\section{DISCUSSION}

The development of the Skalafjord since the last deglaciation was studied by Bennike et al. (1998; macrofossils in core SKPC-18), Jozkow (2000; diatoms in core SKPC-18) and Wachnicka (1999; diatoms in core DAPC-01).

The diatom record in core SKPC-01B provides excellent documentation of the Holocene development of the Faeroe Islands area - the best record of Early Holocene changes known so far. Well-preserved lacustrine deposits at a similar altitude within the Skalafjord were also studied by Jozkow (2000). However, the upper part of core SKPC-18 studied by Jozkow (2000) apparently did not contain the complete record of the diatom flora.

The sediments of core SKPC-01B show two distinct developmental stages. The first one recorded in the lowermost part of the core, subzone DAZ-1a, encompasses lacustrine sediments with a very low content of marine- and brackish-water diatoms. As the proportion between fully marine taxa and brackish-water ones is similar, it is assumed that no permanent connection existed between the central part of the fjord and the ocean. Apparently, diatoms of a marine origin were transported into the Skalafjord during storms. It appears that relative sea-level was below the threshold.

The species composition recorded in subzone DAZ-1b indicates the existence of a lake with abundant planktonic diatoms dominated by $C$. rossii and $F$. ulna (Fig. 4). Their vertical distributions do not show any dramatic changes, implying rather stable conditions. Sporadic inflow of marine waters did not cause any spectacular changes during this developmental stage. Environmental conditions of diatoms in subzone DAZ-1a indicate oligotrophic to mesotrophic waters (Krammer \& Lange-Bertalot, 1986, 1997, 1991a, b, 2000; Håkansson, 1990; Denys, 1992; Hoffman, 1994; Van Dam et al., 1994). It is likely that the dominating taxon, $C$. rossii, formed blooms. Most of the taxa recorded in DAZ-1a are typical for oligotrophic to dystrophic waters (e.g. Lange-Bertalot \& Metzeltin, 1996) accompanied by, for example, $A$. distans, $F$. exigua and the $T$. flocculosa complex (Fig. 4). Generally, within DAZ-1a, a continuous distribution of freshwater taxa is observed. Several of these taxa have been recently described (e.g. Lange-Bertalot \& Metzeltin, 1996; Krammer, 2000, 2002) or are known only from very few localities. e.g. Fragilaria opacolineata Lange-Bertalot, Stauroneis neohyalina Lange-Bertalot, Gomphonema subtile Ehrenberg, Pinnularia ovata Krammer, Pinnularia platycephala Krammer and Pinnularia turbulenta Krammer.

The Skalafjord lake formed after the last deglaciation of the area at c. 10000 years BP (core SKPC-18; Bennike et al., 1998). The diatom record of the early stages of lake development is recorded by Jozkow (2000). The chronology of core SKPC-18 was established from ${ }^{14} \mathrm{C}$ dating and tephra chronology based on the Saksunarvatn ash (Bennike et al., 1998). Diatom analysis of this core revealed a species composition very similar to that in core SKPC-01B, with C. rossii as the dominant species. As core SKPC-01B did not penetrate the Saksunarvatn ash and the uppermost part of the former core is disturbed, these two cores complement each other. Core SKPC-18 provides a record of the early stages of lake development, while core SKPC-01B records the later stage including the transition from lacustrine to marine conditions.

Bennike et al. (1998) determined the inundation of the threshold and the change from lacustrine to marine conditions in core SKPC-18 at c. $7800{ }^{14} \mathrm{C}$ years BP. They also determined the relative sea-level which at that time was $c .25 \mathrm{~m}$ below the present sea-level. However, in core SKPC-01B, the transition between lacustrine and marine conditions is dated between 7700 years BP and 6400 years BP. Previously, studies of the benthic foraminiferal fauna of the fjord (Juul, 1992) indicated that the marine transgression occurred around $7500{ }^{14} \mathrm{C}$ years BP. In addition the diatom species composition prior to the change from lacustrine to marine conditions shows a rise in marine diatoms at $185-180 \mathrm{~cm}$. In the overlying section $(180-165 \mathrm{~cm})$ lacustrine taxa with $C$. rossii dominate again, with a distinct decrease in marine forms.

The effect of a tsunami, triggered by the Storegga Slide, has been documented in Norway and Britain (e.g. Dawson et al., 1988; Bondevik et al., 1997). Deposits resulting from this event were recognized by Grauert et al. (2001) in Lake Vagur on Suduroy Island, which is located south of Skalafjord. The lithology (redeposited organic material and marine microfossils) marks the tsunami section. The age of the tsunami event was estimated at c. 7200 years BP. In core SKPC-01B the rapid increase in marine taxa between $180 \mathrm{~cm}$ and $170 \mathrm{~cm}$ (Fig. 3) may signal an inflow of marine waters. As there is no simultaneous change in lithology it appears that this event did not significantly affect sedimentation processes in the lake. Therefore, this event may instead have been caused by a storm surge. The change in diatom flora at $180-170 \mathrm{~cm}$ in core SKPC-01B may be evidence of one of the first large-scale inflows of marine waters prior to the inundation of the threshold. It may be assumed that the relative sea-level at that time was a few metres lower than $25 \mathrm{~m}$, as limnic conditions were re-established in the basin after termination of the marine inflow. The beginning of environmental change from lacustrine conditions, which resulted in the establishment of the marine environment, is dated between 
$7000{ }^{14} \mathrm{C}$ years $\mathrm{BP}$ and $6400{ }^{14} \mathrm{C}$ years $\mathrm{BP}$, implying that environmental change took place rather rapidly.

Diatom analysis of subzone DAZ-1b shows that the lake in Skalafjord was affected by a strong inflow (possibly catastrophic) of marine waters. This phenomenon happened somewhat later than $7700{ }^{14} \mathrm{C}$ years BP. However, towards the top of this subzone lacustrine conditions were re-established. The development of the lake in the Skalafjord implies that the Storegga Slide led to inflow of marine waters at a distinctly lower water level in the area of Eysturoy and that the inundation of the threshold in the fjord happened after the tsunami. The lithology of this part of the core indicates that the tsunami impact in this area was relatively weak.

\section{ACKNOWLEDGEMENTS}

We wish to express our gratitude to Björn Malmgren, University of Göteborg, Ole Bennike and Antoon Kuijpers, Geological Survey of Denmark and Greenland, Copenhagen, for their critical comments, support and improvement of the text. Special thanks are due to Ditmar Metzeltin and Horst Lange-Bertalot for providing detailed comments on the diatom taxonomy.

\section{APPENDIX A: TAXONOMIC NOTE}

Either the dominating or interesting taxa are listed below and the sources of their identification are given. The identification was primarily based on the diatom flora of middle Europe by Krammer \& Lange-Bertalot (1986, 1991a, b, 1997). The reason for choosing this was that all the taxa treated in these references are illustrated with relevant LM micrographs. For the complete list of taxa see Appendix B.

Achnanthes holstii Cleve

Lit.: Krammer \& Lange-Bertalot (1991b, p. 33, fig. 18: 14-17)

P1. 3, fig. 16

Achnanthes pusilla (Grunow) de Toni

Syn.: Achnanthes (linearis var.?) pusilla Grunow in Cleve \& Grunow

Lit.: Krammer \& Lange-Bertalot (1991b, p. 67, fig. 37: 9-18)

Pl. 3, figs 11-13

Amphora inariensis Krammer

Lit.: Krammer \& Lange-Bertalot (1986, p. 310, fig. 96: 18-20)

Pl. 5, figs 10-12

Amphora veneta Kützing

Lit.: Krammer \& Lange-Bertalot (1986, p. 348, fig. 151: 7-17)

Pl. 5, figs 8, 9

Aneumastus rostratus (Hustedt) Lange-Bertalot

Syn.: Navicula tuscula var. rostrata Hustedt; Navicula tusculoides Cleve-Euler

Lit.: Lange-Bertalot (2001, p. 156, fig. 118: 1-6)

Pl. 5, fig. 18

Aulacoseira distans (Ehrenberg) Simonsen

Syn.: Gallionella distans Ehrenberg; Melosira distans (Ehrenberg) Kützing

Lit.: Krammer \& Lange-Bertalot (1991a, p. 32, fig. 29: 1-23, fig. 30: 1-11)

Pl. 1, figs 6-11
Aulacoseira italica (Ehrenberg) Simonsen

Syn.: Gallionella italica Ehrenberg; Melosira italica (Ehrenberg) Kützing

Lit.: Krammer \& Lange-Bertalot (1991a, p. 29, fig. 24: 1, 3-6, fig. 25: 1-11)

Pl. 1, fig. 21

Aulacoseira subarctica (O. Müller) Haworth

Syn.: Melosira italica ssp. subarctica O. Müller; Aulacoseira italica ssp. subarctica (O. Müller) Simonsen

Lit.: Krammer \& Lange-Bertalot (1991a, p. 28, fig. 23: 1-11, fig. 2: 1)

Pl. 1, figs 16,17

Aulacoseira valida (Grunow) Krammer

Syn.: Melosira crenulata var. valida Grunow in Van Heurck. Melosira italica var. valida (Grunow) Hustedt. Aulacoseira italica var. valida (Grunow) Simonsen

Lit.: Krammer \& Lange-Bertalot (1991a, p. 32, fig. 28: 1-11) Pl. 1, figs $18-20$

Brachysira brebissonii Ross

Bas.: Navicula aponina var. brachysira Brébisson ex Kützing Syn.: Anomoeoneis brachysira (Brébisson ex. Rabenhorst) Grunow in Cleve

Lit.: Lange-Bertalot \& Moser (1994, p. 20, fig. 12: 6, fig. 41: 1-18, fig. 44: 1-10)

Pl. 12, fig. 9

Brachysira zellensis (Grunow) Round \& Mann

Bas.: Navicula zellensis Grunow

Syn.: Anomoeoneis zellensis (Grunow) Cleve; Anomoeoneis brachysira var. zellensis (Grunow) Krammer

Lit.: Lange-Bertalot \& Moser (1994, p. 73, fig. 11: 24-28, fig. 12: 5)

Pl. 12, fig. 10

Caloneis cf. bacillum (Grunow) Cleve

Syn.: Stauroneis bacillum Grunow; Navicula fasciata Lagerstedt; Caloneis fasciata (Lagerstedt) Cleve; (?)Caloneis bacillaris (Gregory) Cleve

Lit.: Krammer \& Lange-Bertalot (1986, p. 390, fig. 174: 9-20)

Pl. 10, figs 10, 11

Caloneis pulchra Messikommer

Lit.: Krammer \& Lange-Bertalot (1986, p. 392, fig. 173: 1-4) Pl. 10, fig. 14

Caloneis tenuis (Gregory) Krammer

Syn.: Pinnularia tenuis Gregory; Pinnularia gracillima Gregory

Lit.: Krammer \& Lange-Bertalot (1986, p. 392, fig. 174: 5-10) Pl. 10, fig. 13

Caloneis undulata (Gregory) Krammer Syn.: Pinnularia undulata Gregory Lit.: Krammer \& Lange-Bertalot (1986, p. 394, fig. 175: 1-6) Pl. 10, fig. 12

Ceratoneis arcus (Ehrenberg) Kützing var. arcus Syn.: Fragilaria arcus var. arcus (Ehrenberg) Cleve; Ceratoneis amphioxys Rabenhorst; Ceratoneis arcus var. amphioxys (Rabenhorst) Brun; Ceratoneis arcus var. linearis 
Diatoms from Holocene freshwater sediments, Faeroe Islands
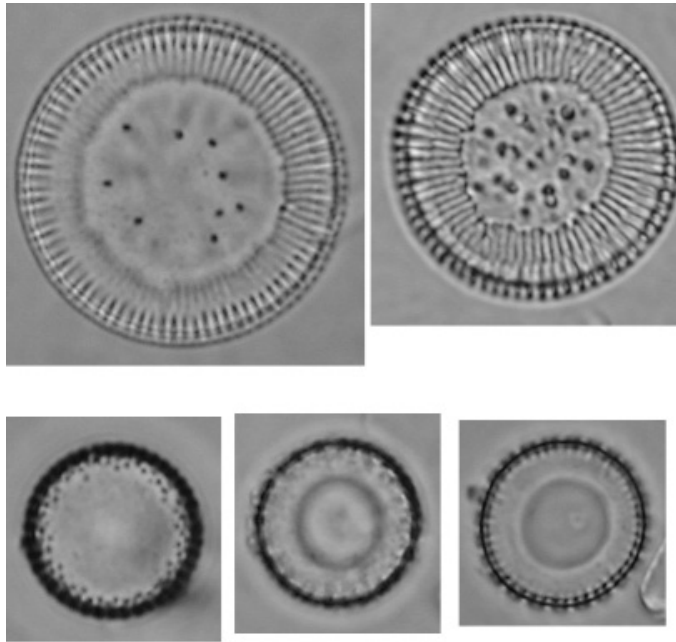

$6-10$
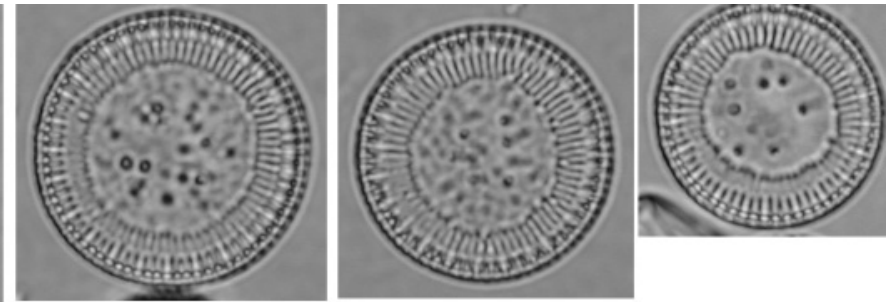

1 - 5
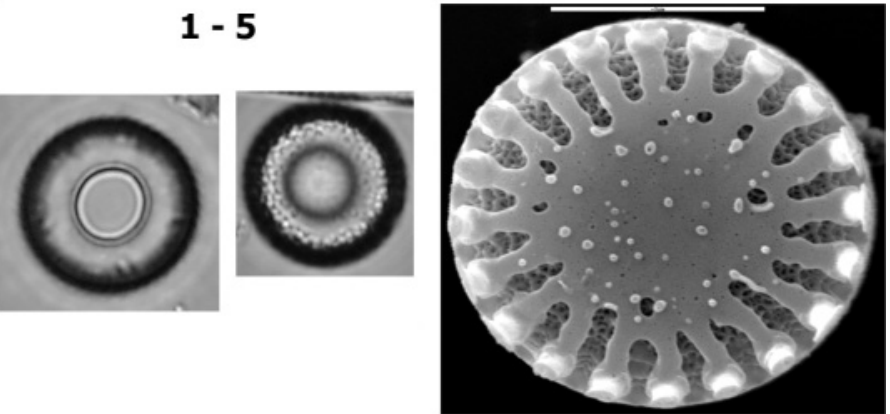

11

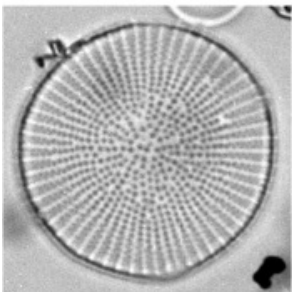

12

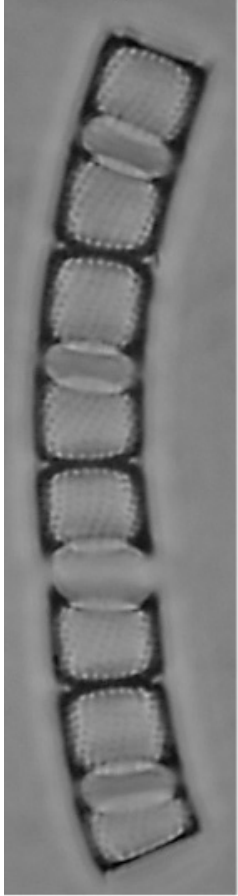

16

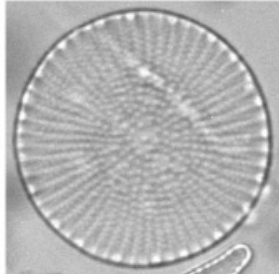

13

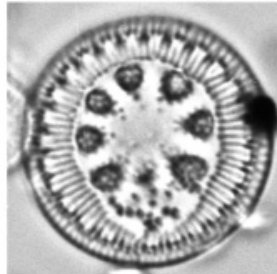

14

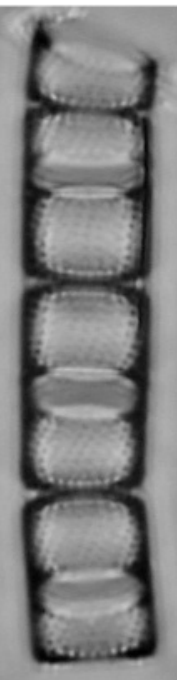

17

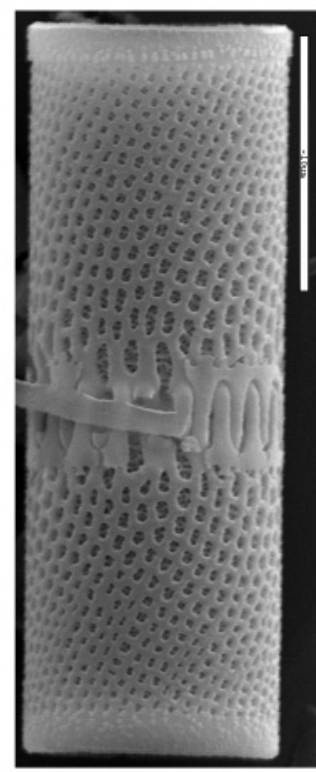

18

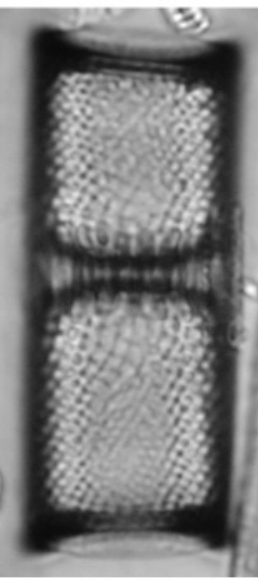

19

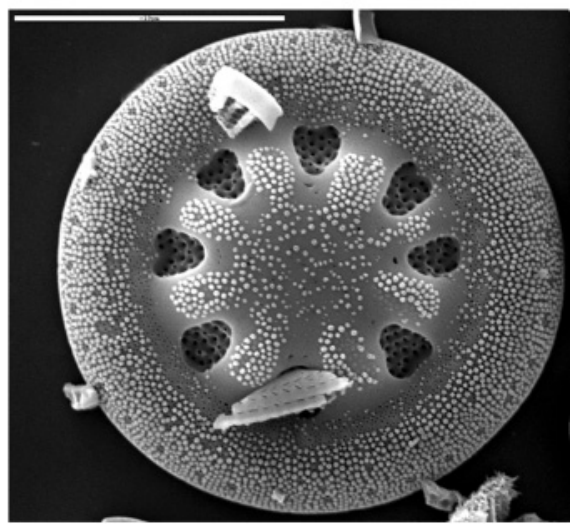

15

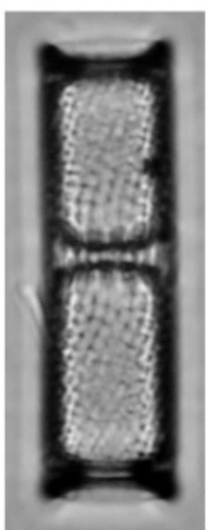

20

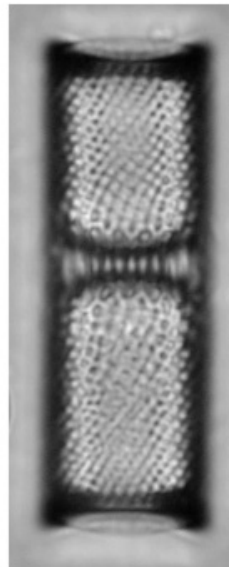

21

Explanation of Plate 1.

figs 1-5. Cyclotella rossii Håkansson. figs 6-11. Aulacoseira distans (Ehrenberg) Simonsen. figs 12, 13. Cyclostephanus invisitatus (Hohn \& Hellerman) Theriot, Stoermer \& Håkansson. figs 14, 15. Cyclotella antiqua W. Smith. figs 16, 17. Aulacoseira subarctica (O. Müller) Haworth. figs 18-20. Aulacoseira valida (Grunow) Krammer. fig. 21. Aulacoseira italica (Ehrenberg) Simonsen. figs 1-10, 12-14, 16-17, 19-21 LM micrographs (magnification $\times 1500$ ); figs $11,15,18$ SEM micrographs (scale bars: $11=5 \mu \mathrm{m} ; 15,18=10 \mu \mathrm{m}$ ). 


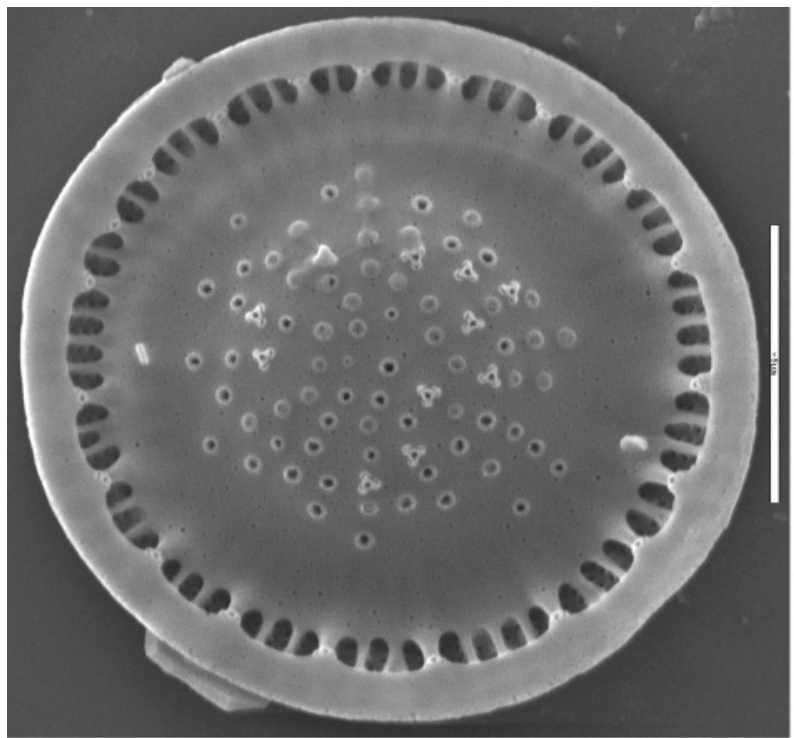

1

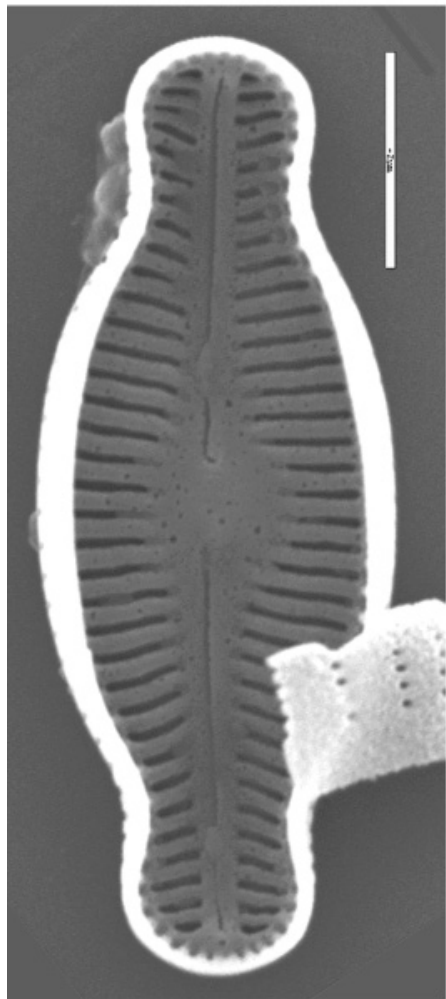

5

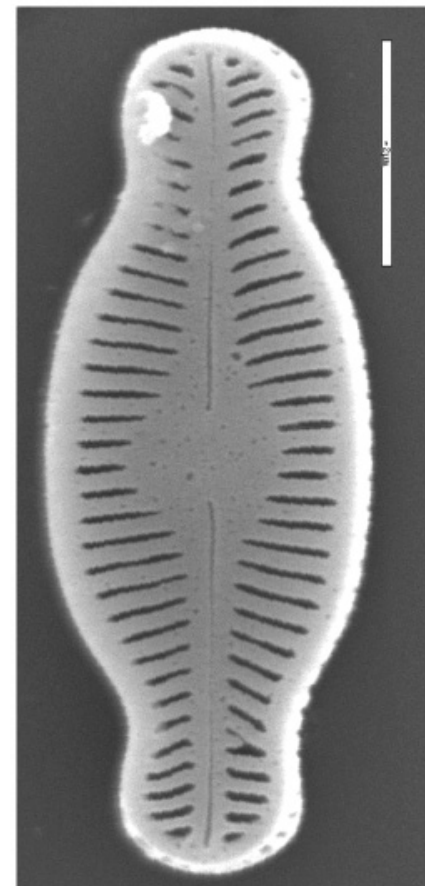

6

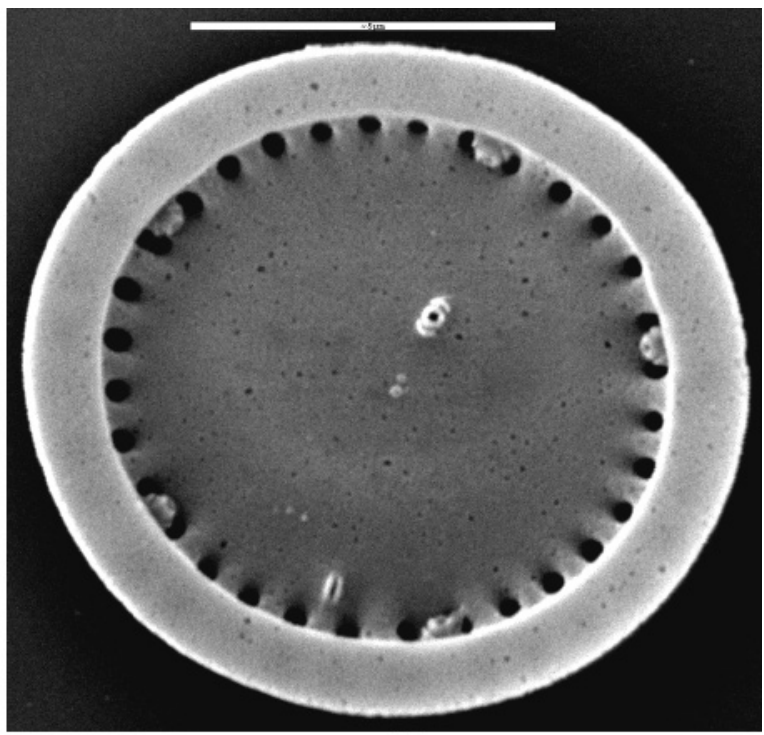

2

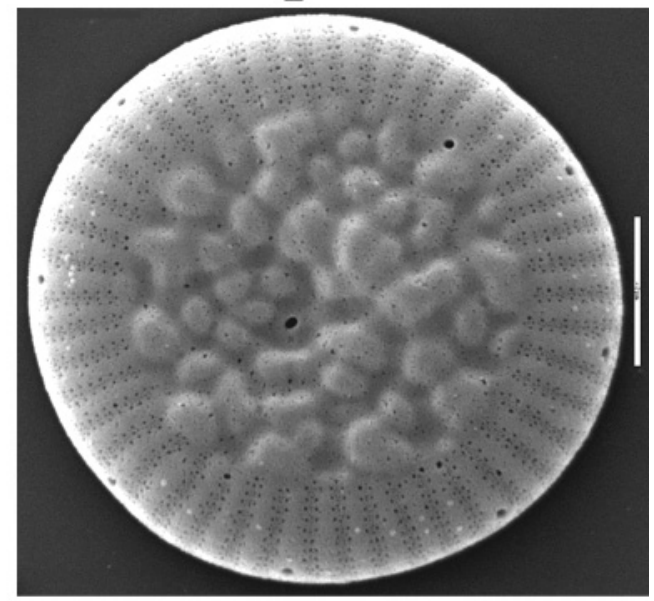

3

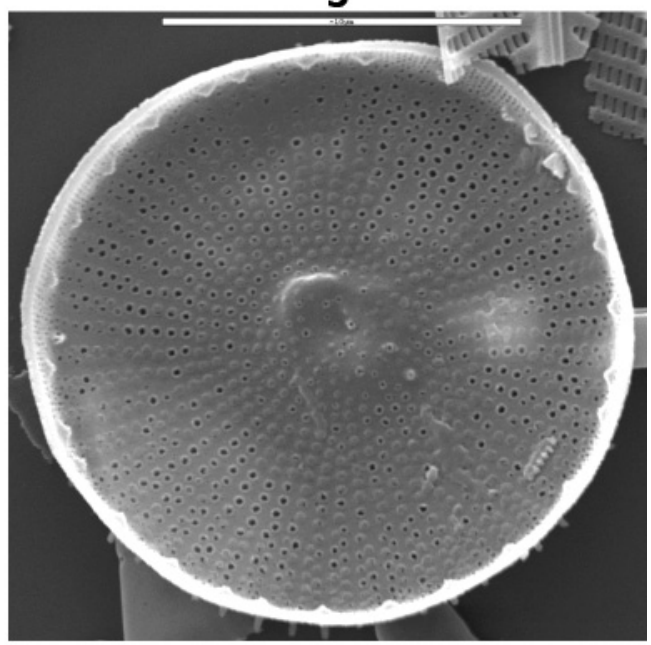

4

Explanation of Plate 2.

fig. 1. Cyclotella radiosa (Grunow) Lemmermann. figs 2, 3. Cyclotella rossi Håkansson. fig. 4. Cyclostephanus invisitatus (Hohn \& Hellerman) Theriot, Stoermer \& Håkansson. figs 5, 6. Navicula schmasmannii Hustedt. All SEM micrographs (scale bars: 1, 2=5 $\mu \mathrm{m} ; 3,5,6=2 \mu \mathrm{m} ; 4=10 \mu \mathrm{m}$ ). 
Diatoms from Holocene freshwater sediments, Faeroe Islands
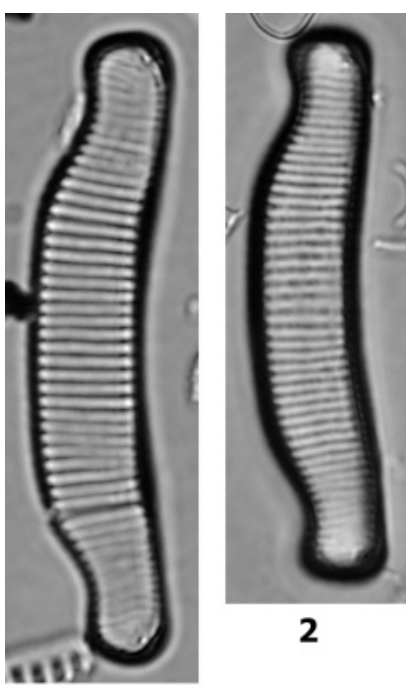

2

1

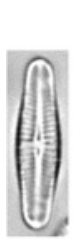

10

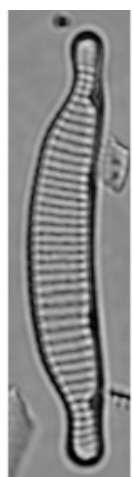

3

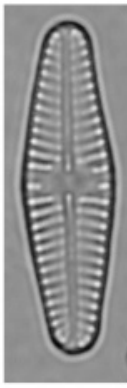

15
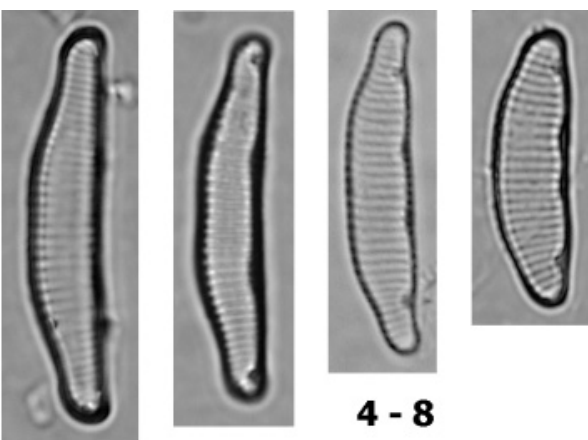

4 - 8

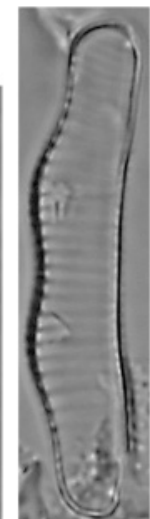

17

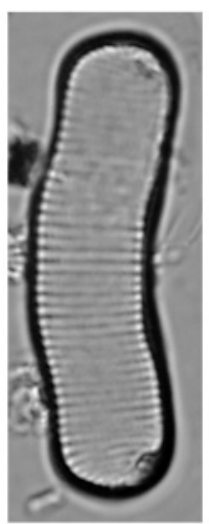

18
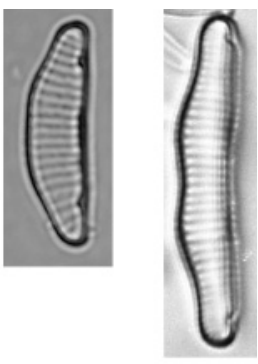

9

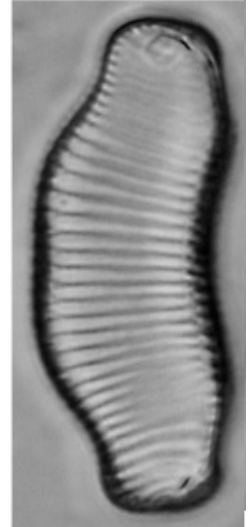

19

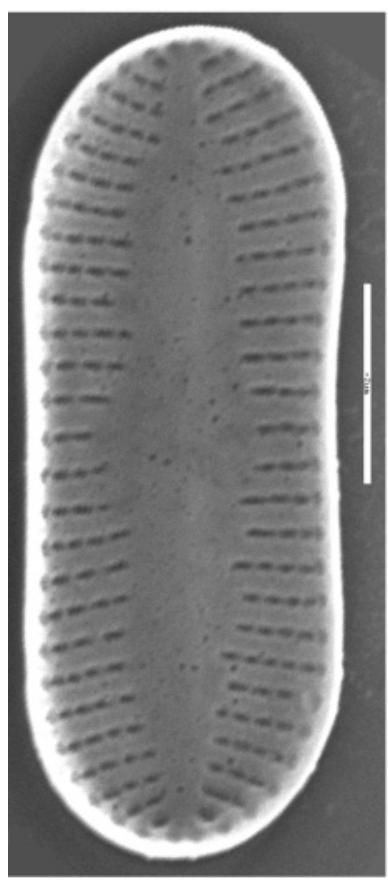

20

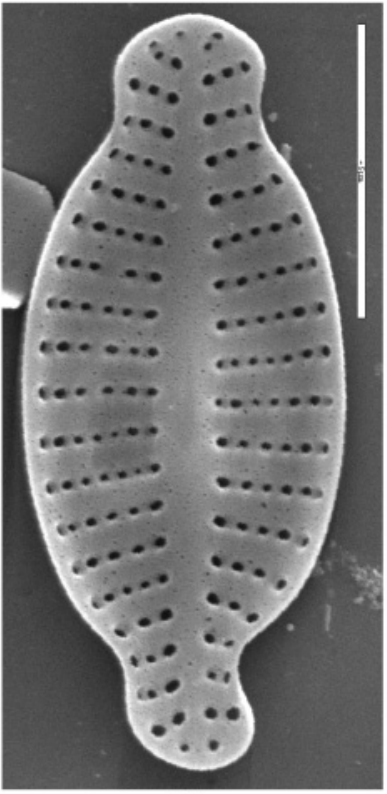

21

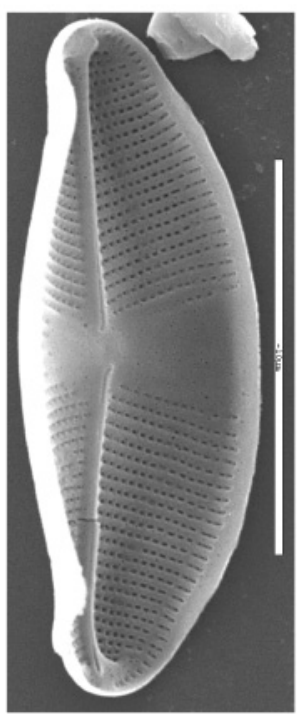

22

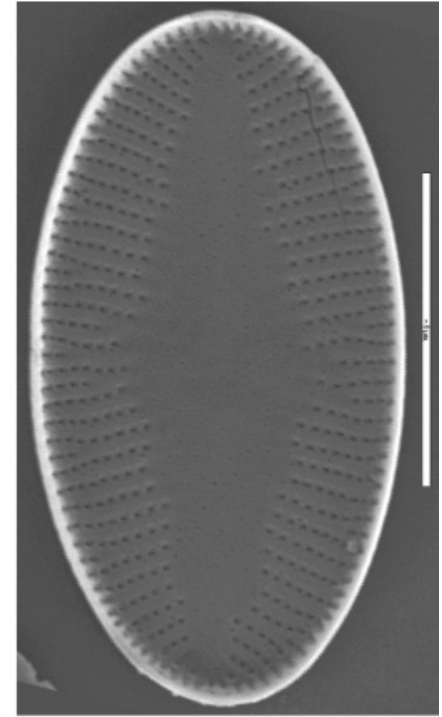

23

Explanation of Plate 3.

figs 1, 2. Eunotia arcus Ehrenberg. fig. 3. Eunotia boreoalpina Lange-Bertalot \& Nörpel-Schempp. figs 4-8. Eunotia incisa Gregory. fig. 9. (?)Eunotia implicata Nörpel-Schempp, Lange-Bertalot \& Alles. fig. 10. Achnanthes minutissima sensu auct. nonnull. figs 11-13. Achnanthes pusilla (Grunow) De Toni. figs 14, 15. Achnanthes lanceolata (Brébisson) Grunow. fig. 16. Achnanthes holstii Cleve. fig. 17. Eunotia circumborealis Nörpel-Schempp \& Lange-Bertalot. fig. 18. Eunotia media A. Cleve. fig. 19. Eunotia praerupta Ehrenberg. fig. 20. Achnanthes didyma Hustedt. fig. 21. Achnanthes laterostrata Hustedt. fig. 22. Eucocconeis laevis (Østrup) Lange-Bertalot. fig. 23. (?)Achnanthes daonensis Lange-Bertalot. figs 1-19 LM micrographs (magnification $\times 1500$ ); figs $20-23$ SEM micrographs (scale bars: $20=2 \mu \mathrm{m} ; 21,23=5 \mu \mathrm{m} ; 22=10 \mu \mathrm{m}$ ). 
E. Witon \& A. Witkowski

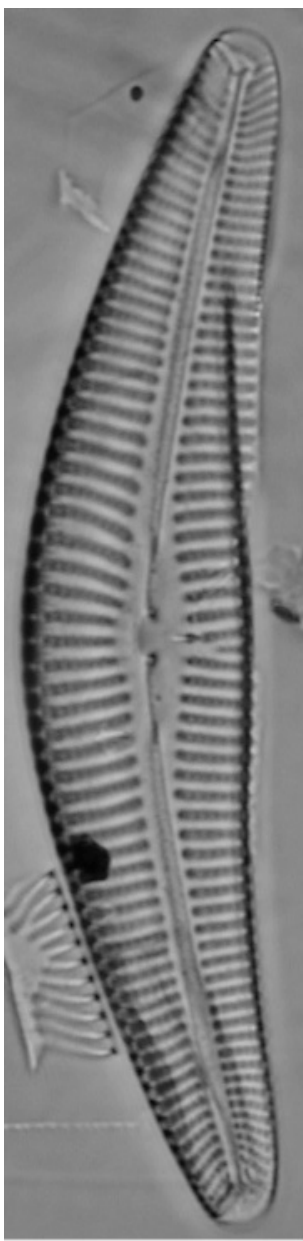

1

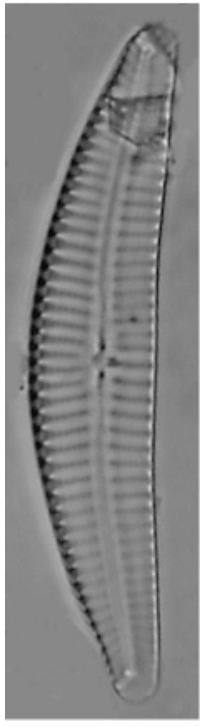

6

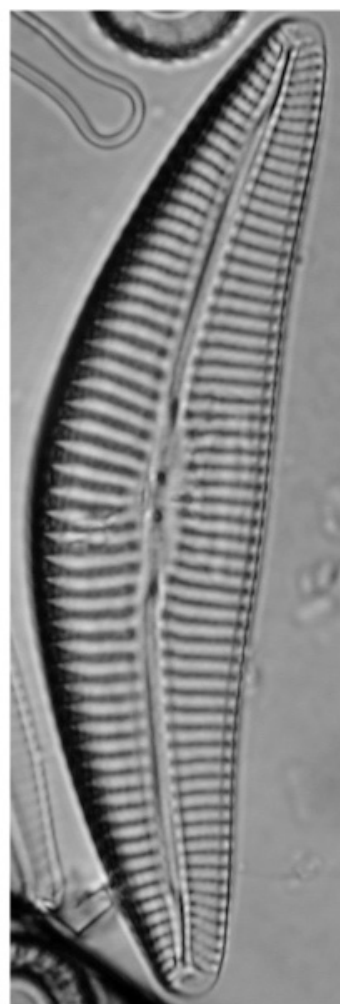

2

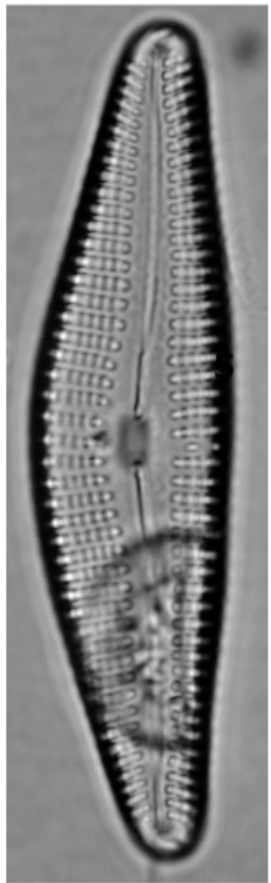

7

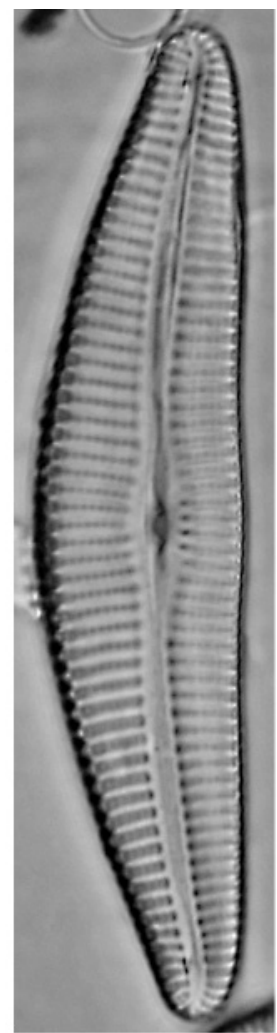

3

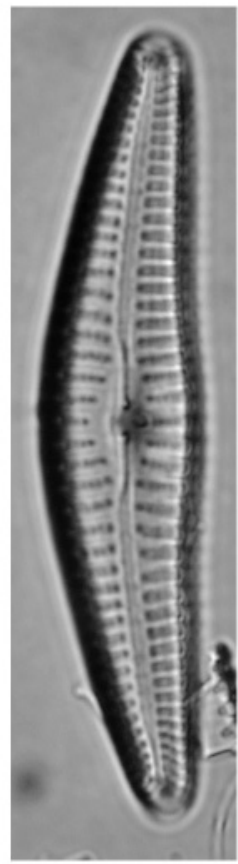

8

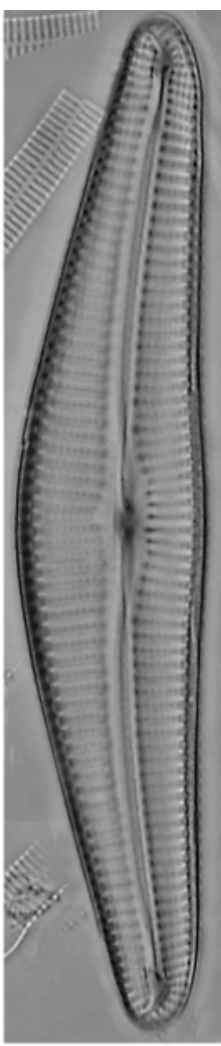

4

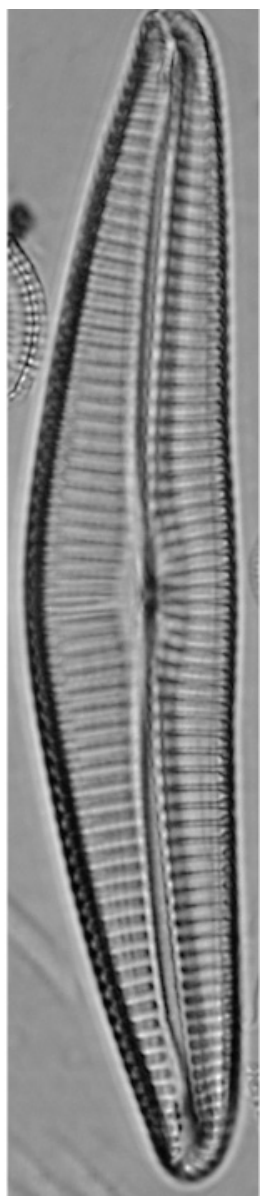

5

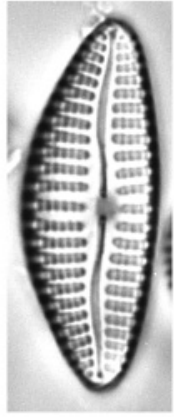

9

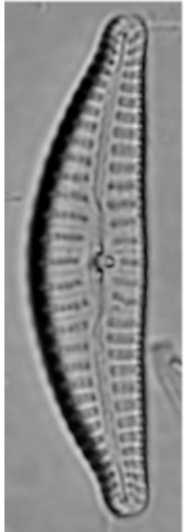

10

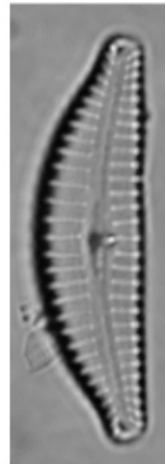

11

Explanation of Plate 4.

figs 1, 2. Cymbella spec. cf. helvetica Kützing. figs 3-5. Cymbella lange-bertalotii Krammer. fig. 6. Cymbella vulgata Krammer. figs 7, 8. Cymbella neoleptoceros var. tenuistriata Krammer. fig. 9. Cymbella pervarians Krammer. figs 10, 11. Cymbella cf. subtruncata Krammer. All LM micrographs (magnification $\times 1500)$. 
Diatoms from Holocene freshwater sediments, Faeroe Islands

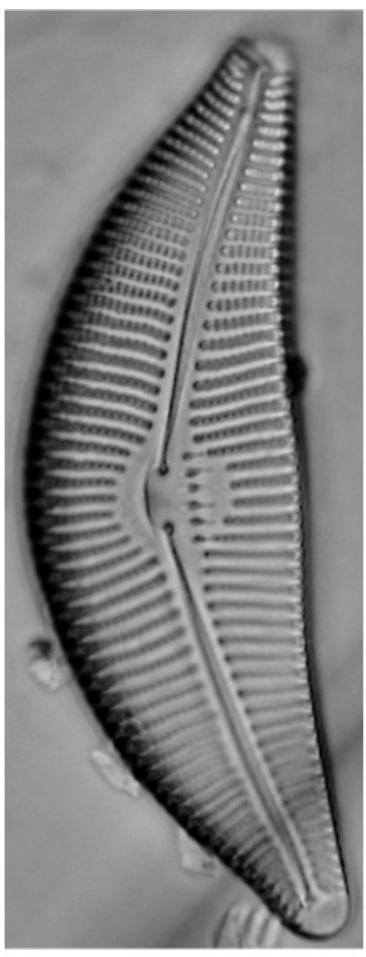

1
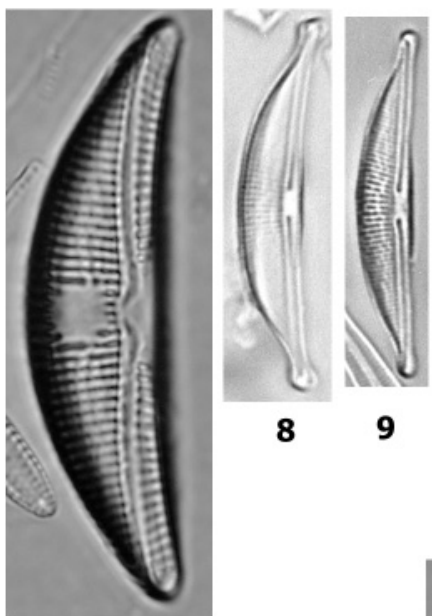

7
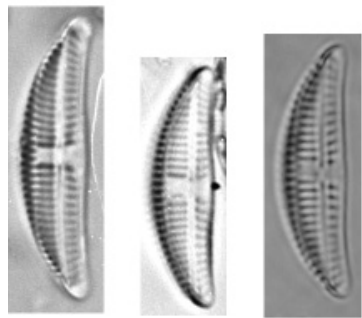

$10-12$

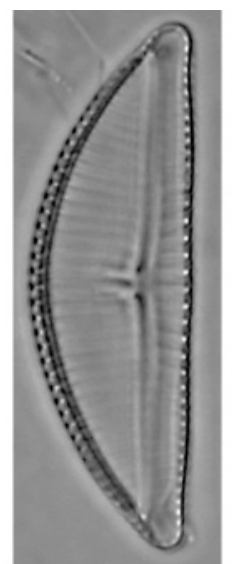

2

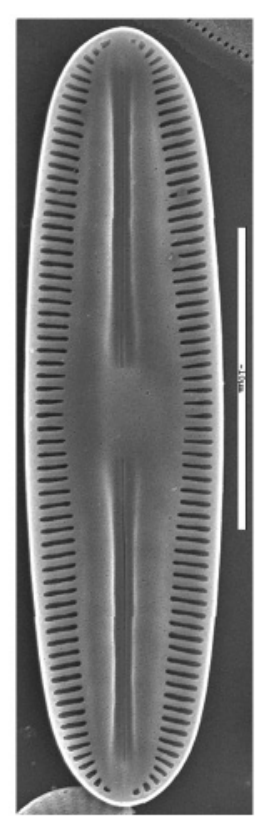

13
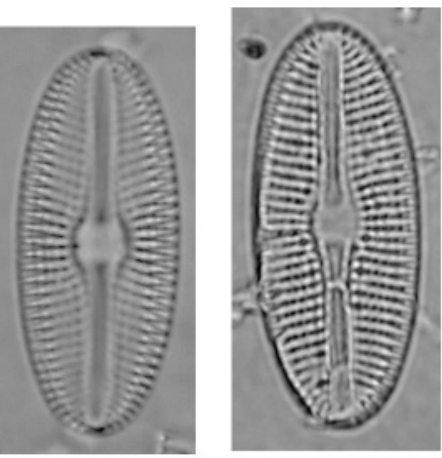

14 - 16

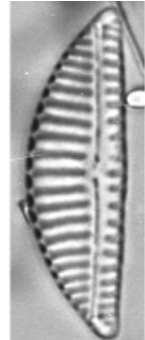

4
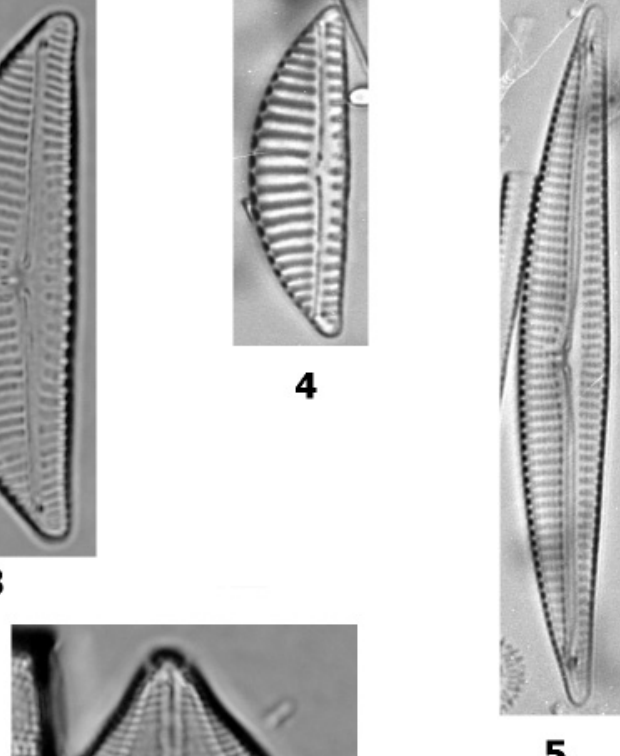

5

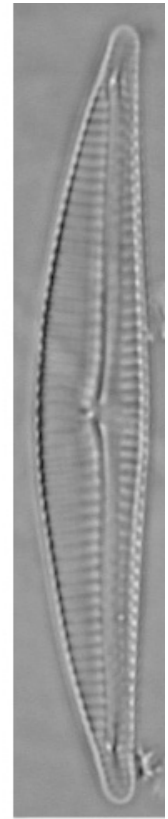

6
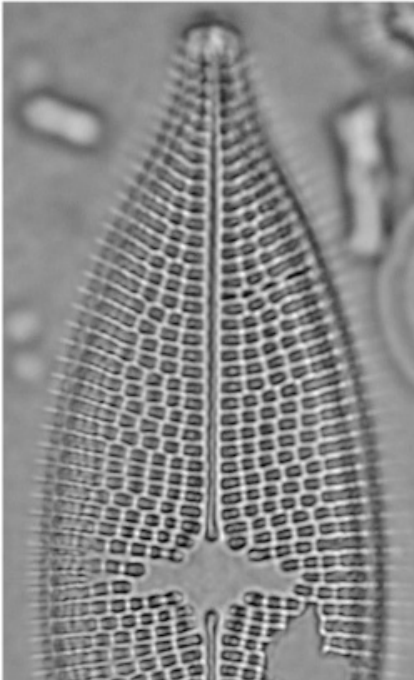

17
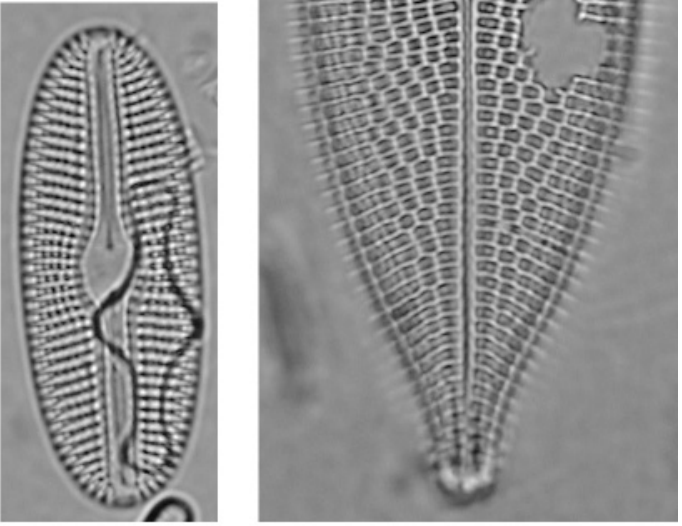

18

Explanation of Plate 5.

fig. 1. Cymbella proxima Reimer. figs 2, 3. Encyonema silesiacum (Bleisch) D.G. Mann. fig. 4. Encyonema procerum Krammer. figs 5, 6. Encyonema neogracile Krammer. fig. 7. Amphora copulata (Kützing) Schoeman \& Archibald. figs 8, 9. Amphora veneta Kützing. figs 10-12. Amphora inariensis Krammer. fig. 13. Diploneis petersenii Hustedt. figs 14-16. Diploneis pseudovalis Hustedt. fig. 17. Neidium apiculatum Reimer. fig. 18. Aneumastus rostratus Lange-Bertalot. figs 1-12, 14-18 LM micrographs (magnification $\times 1500$ ); fig. 13 SEM micrograph (scale bar: $10 \mu \mathrm{m})$. 
E. Witon \& A. Witkowski

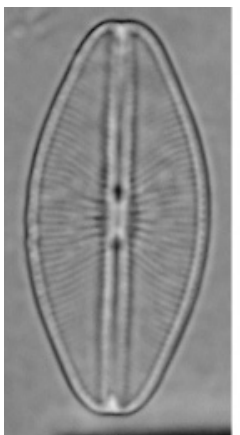

1

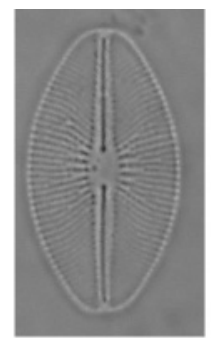

2

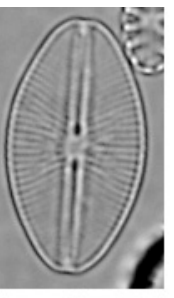

3
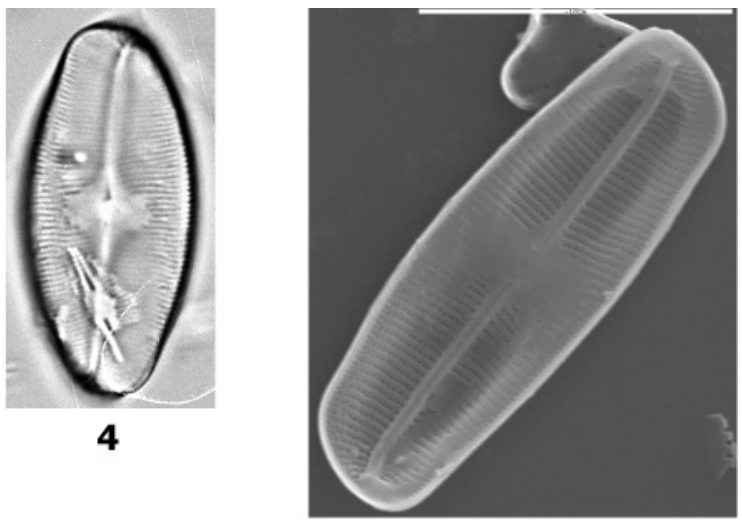

5

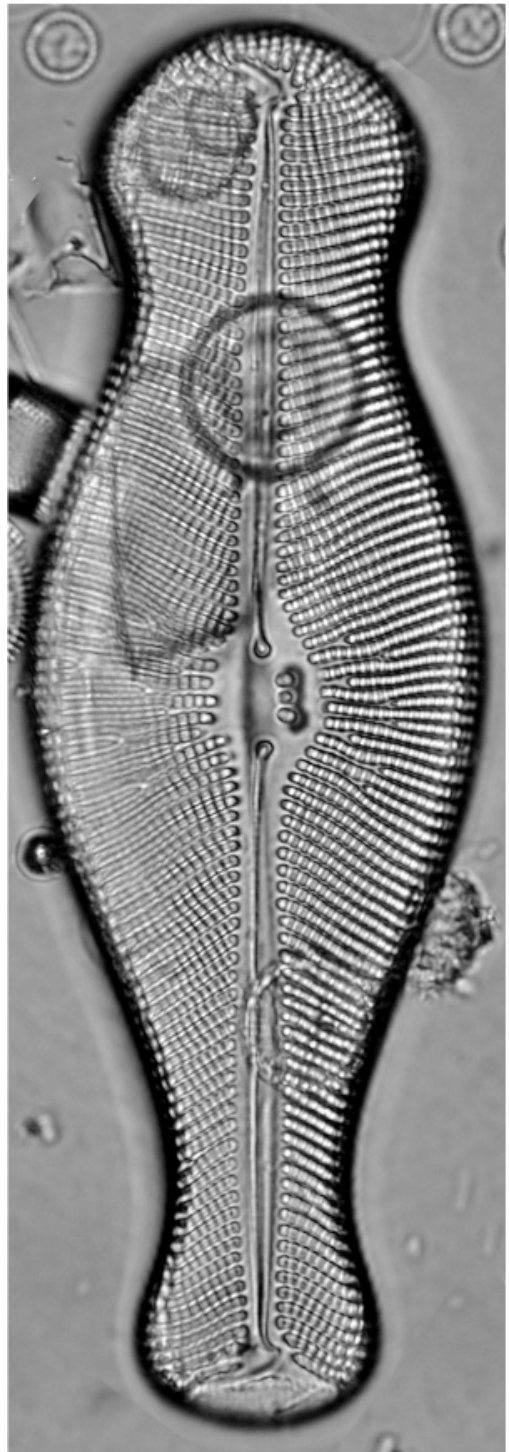

6

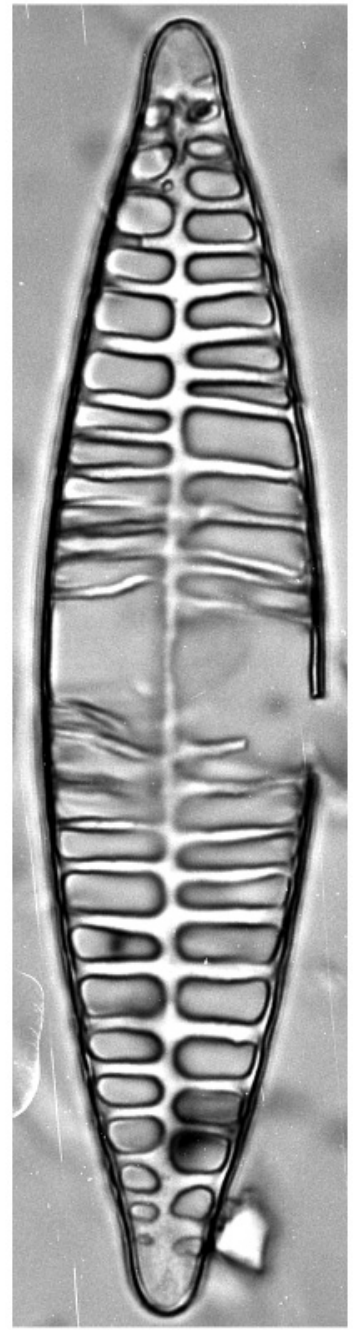

7

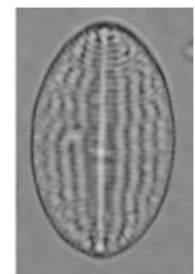

8

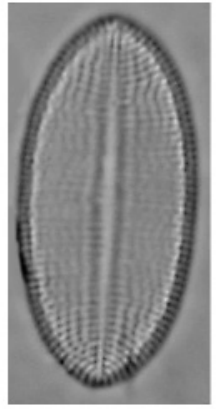

9

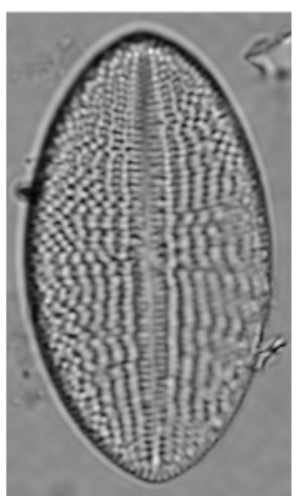

10

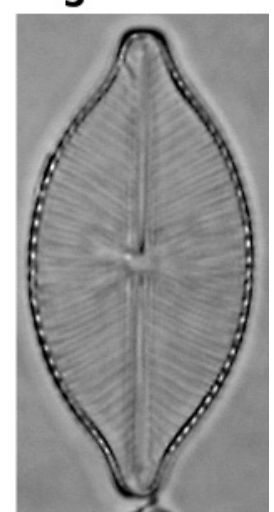

11

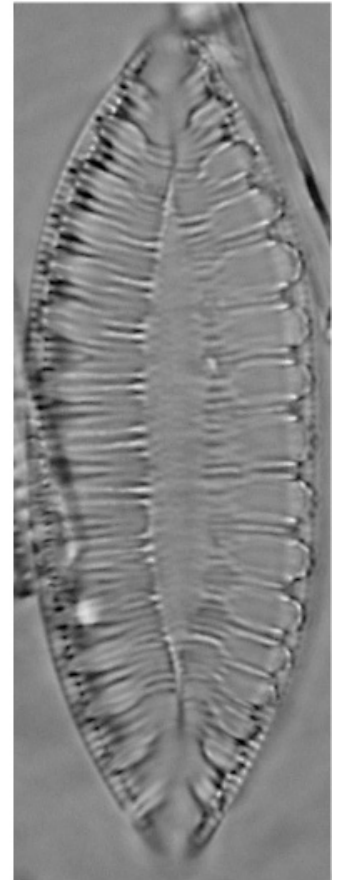

12

Explanation of Plate 6.

figs 1-3. Cavinula cocconeiformis (Gregory) D.G. Mann \& Stickle. fig. 4. Eucocconeis flexella (Kützing) Cleve var. flexella. fig. 5. Eucocconeis alpestris (Brun) Lange-Bertalot. fig. 6. Didymosphenia geminata (Lyngbye) M. Schmidt, Morphotyp geminata sensu Metzeltin \& Lange-Bertalot (1995). fig. 7. Craticula cuspidata (Kützing) D. G. Mann. figs 8-10. Cocconeis placentula var. lineata (Ehrenberg) Van Heurck. fig. 11. Placoneis cf. clementis (Grunow). fig. 12. Surirella amphioxys W. Smith. figs 1-4, 6-12 LM micrographs (magnification $\times 1500$ ); fig. 5 SEM micrograph (scale bar: $10 \mu$ m). 
Diatoms from Holocene freshwater sediments, Faeroe Islands
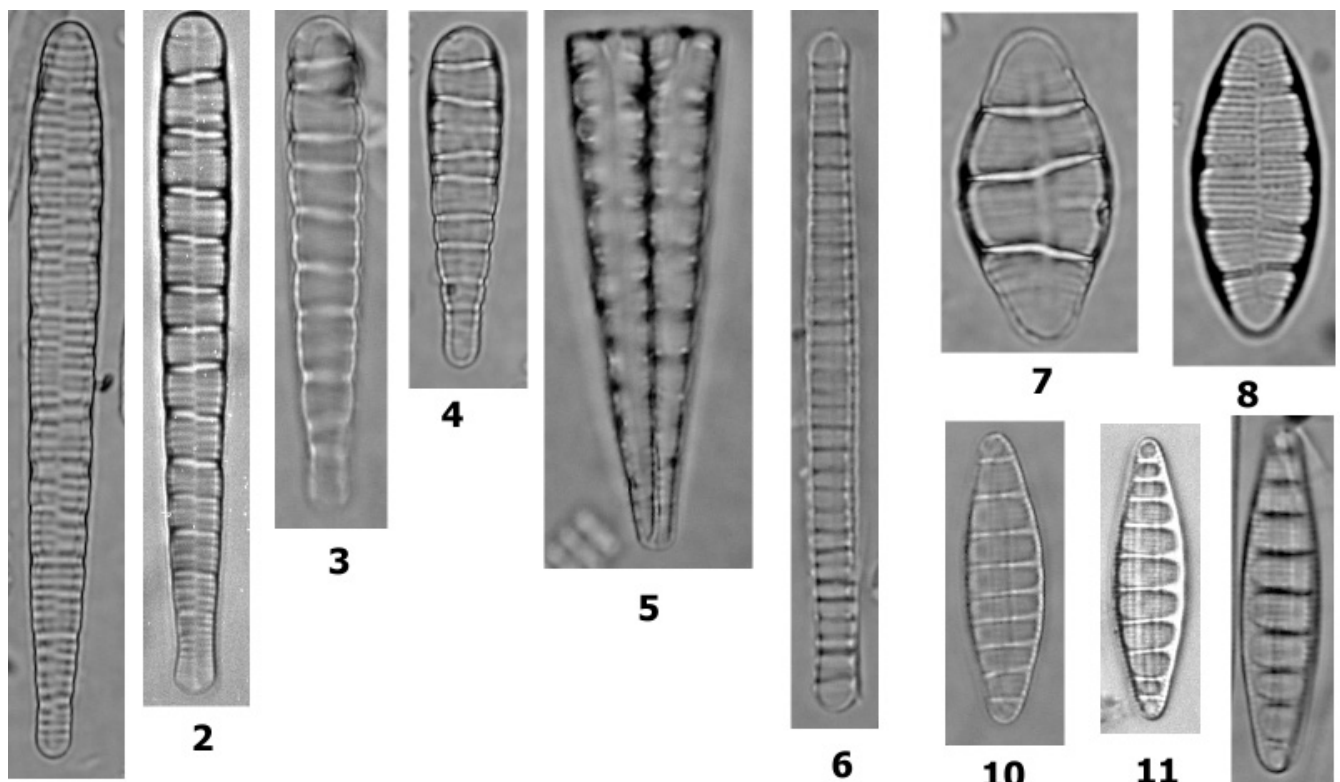

1

5

7

8

6
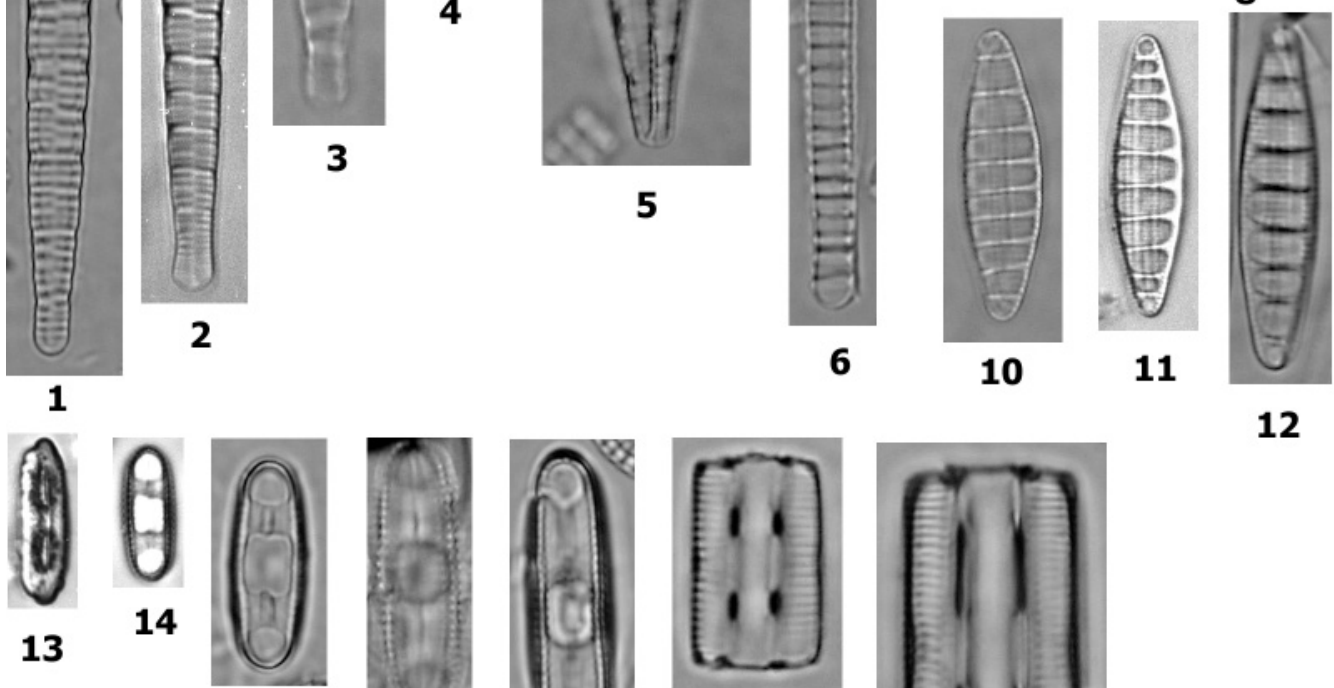

15
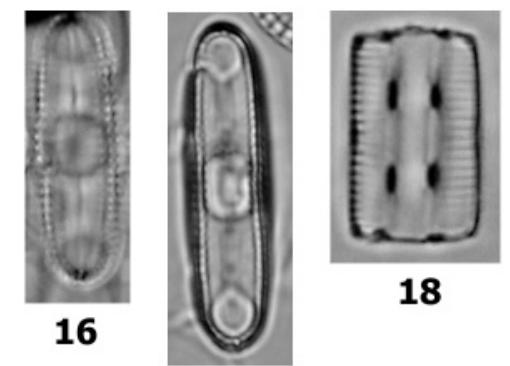

18
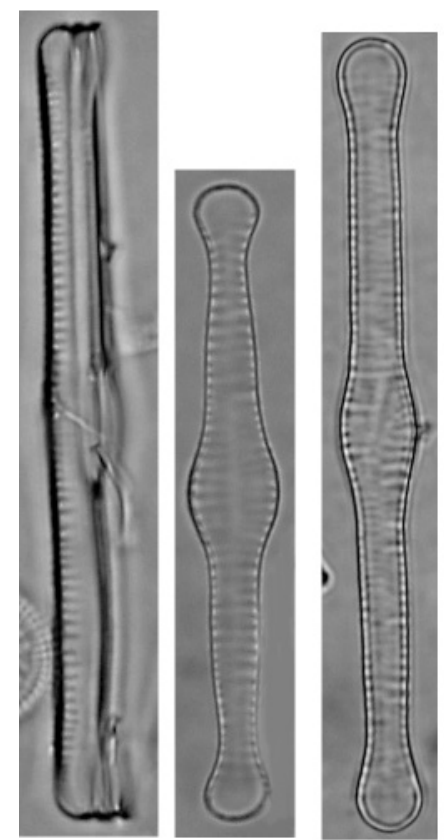

$20-23$
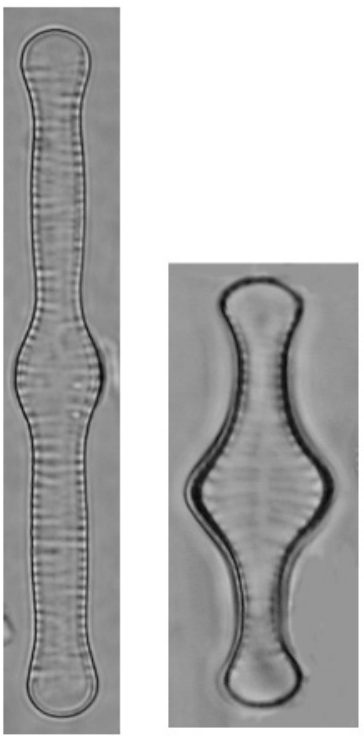

24

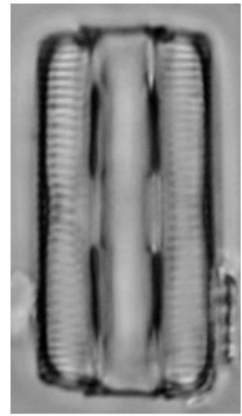

19
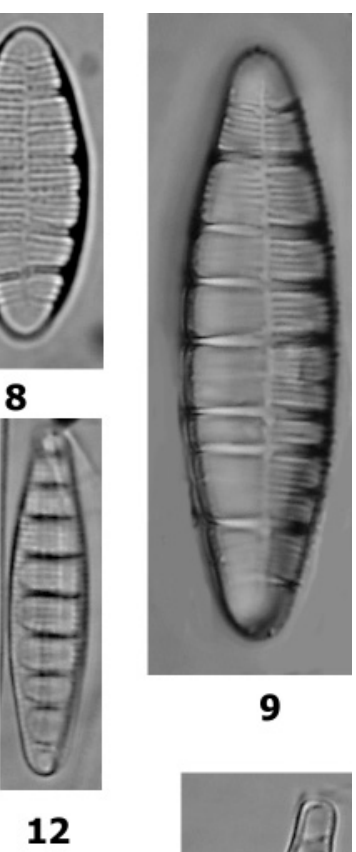

9

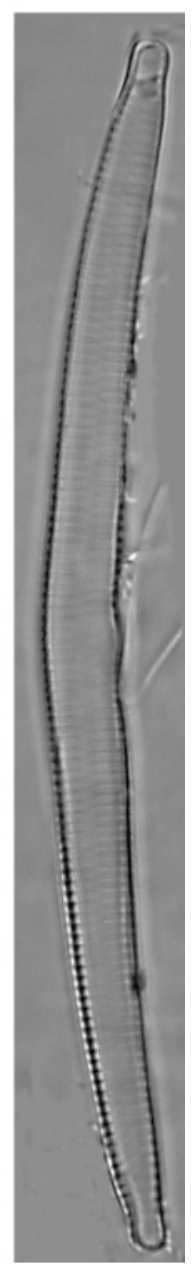

26 - 28

Explanation of Plate 7.

figs 1-5. Meridion circulare (Greville) Agardh. fig. 6. Diatoma tenuis Agardh. figs 7-9. Diatoma mesodon (Ehrenberg) Kützing. figs 10-12. Denticula tenuis Kützing. figs 13-19. Diatomella balfouriana Greville. figs 20-23. Tabellaria flocculosa sensu lato (Roth) Kützing. figs 24, 25. Tabellaria flocculosa sensu stricto (Roth) Kützing. figs 26-28. Ceratoneis arcus (Ehrenberg) Kützing var. arcus. All LM micrographs (magnification $\times 1500$ ). 
E. Witon \& A. Witkowski
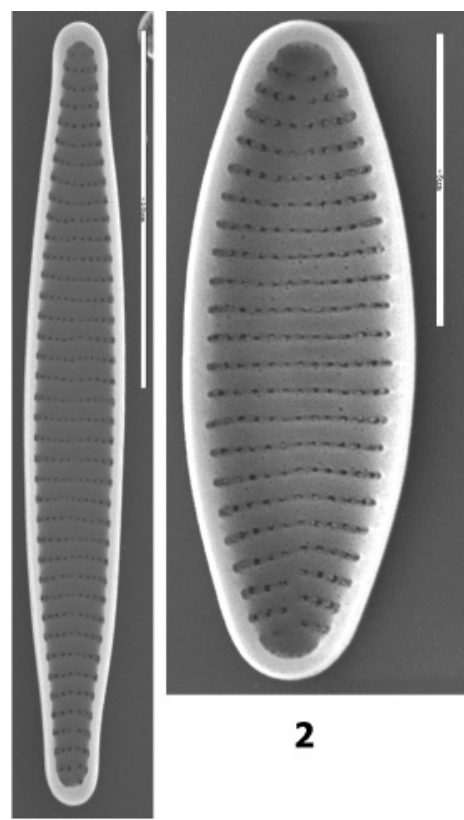

2

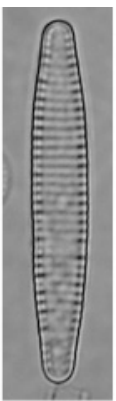

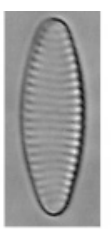

3- 5

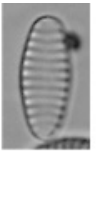

5

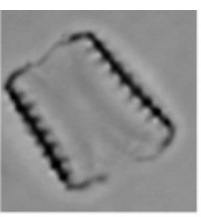

6

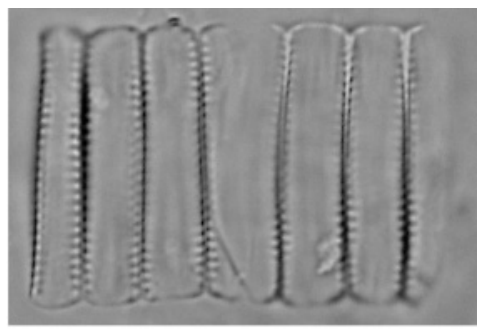

7
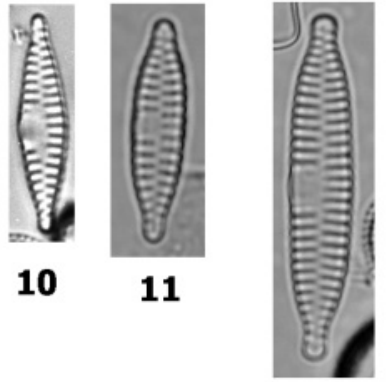

12

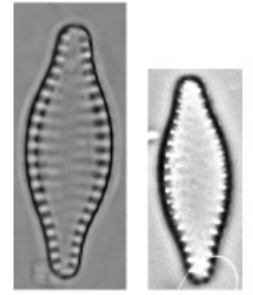

15 - 17
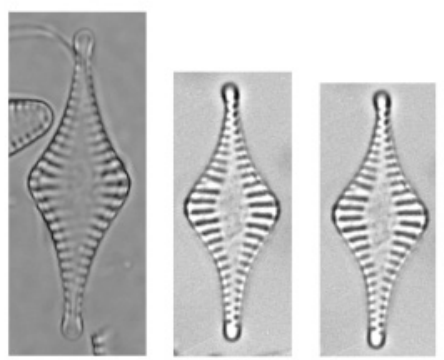

$18-20$

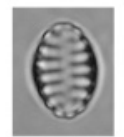

21

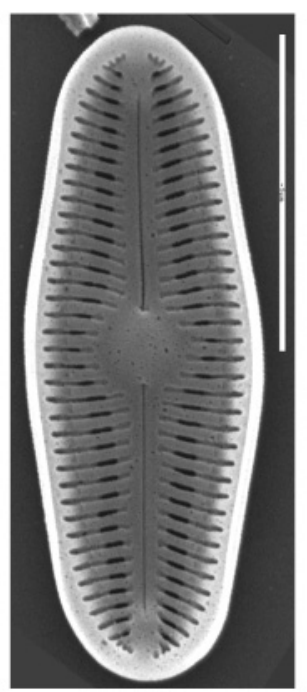

32

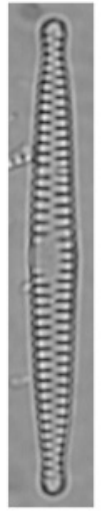

13

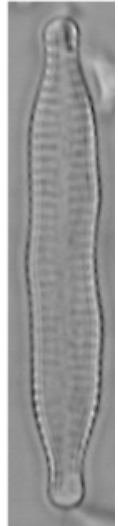

14

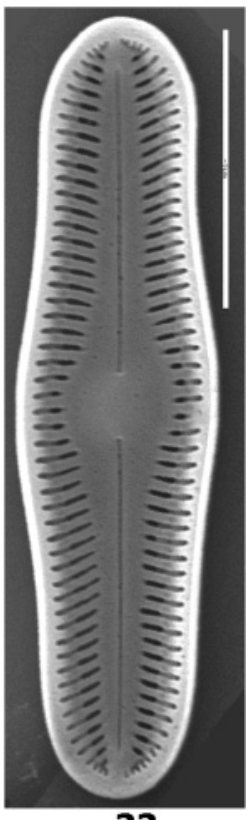

33

Explanation of Plate 8.

figs 1-5, 7. Fragilaria exigua Grunow. fig. 6. Staurosira martyi (Héribaud) Bukhtiyarova. figs 8, 9. Fragilaria construens var. binodis fo. borealis? Foged. figs 10, 11. Fragilaria capucina var. perminuta (Grunow) Lange-Bertalot. fig. 12. Fragilaria capucina var. vaucheriae (Kützing) Lange-Bertalot. fig. 13. Fragilaria capucina var. distans (Grunow) Lange-Bertalot. fig. 14. Fragilaria alpestris Krasske. figs 15-17. Fragilaria opaclineolata Lange-Bertalot. figs 18-20. Fragilaria parasitica (W. Smith) Grunow. fig. 21. Staurosira construens (Ehrenberg) Grunow. figs 22-24. Staurosira pseudoconstruens (Marciniak) Lange-Bertalot. figs 25-27. Staurosira sp.. figs 28, 29. Diadesmis biceps Arnott. figs 30-33. Diadesmis perpusilla (Grunow) Lange-Bertalot. figs 3-24, 26-31 LM micrographs (magnification $\times 1500$ ); figs 1, 2, 25, 32, 33 SEM micrographs (scale bars: 1=10 $\mu$; 2 , $25,32,33=5 \mu \mathrm{m})$. 
Diatoms from Holocene freshwater sediments, Faeroe Islands
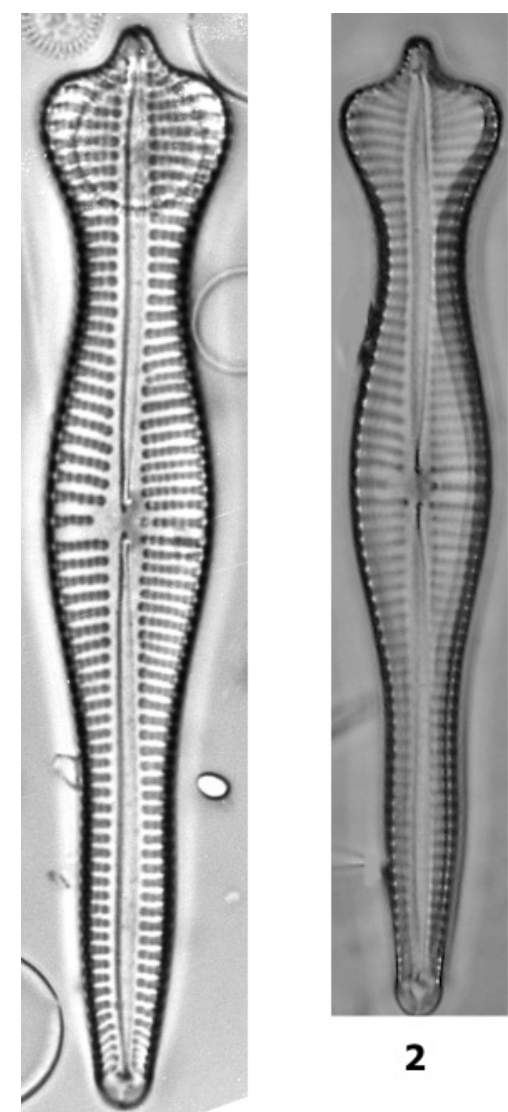

2

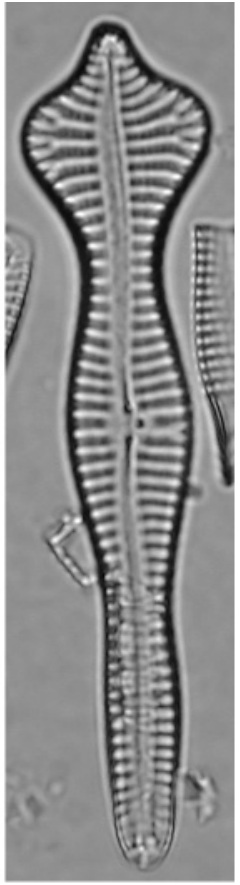

3

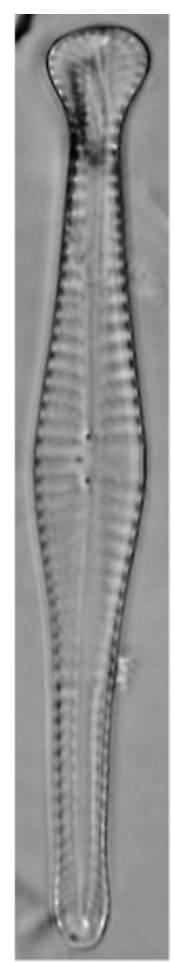

4

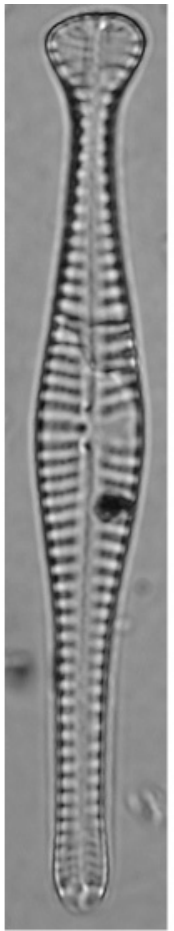

5

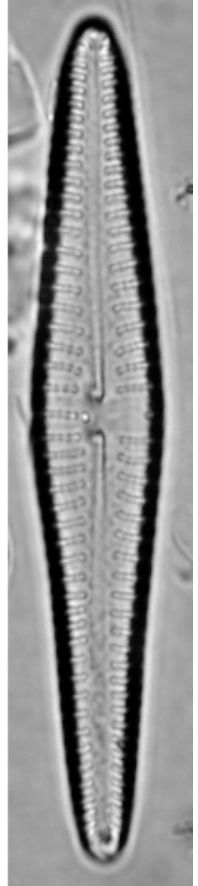

6

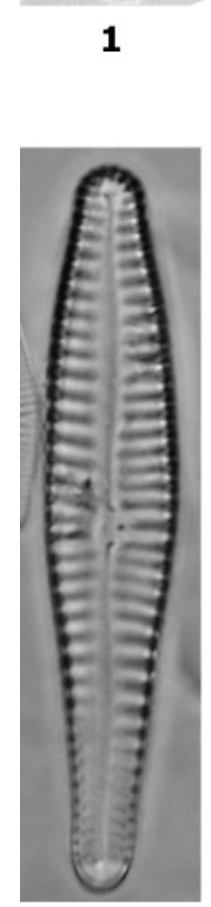

9

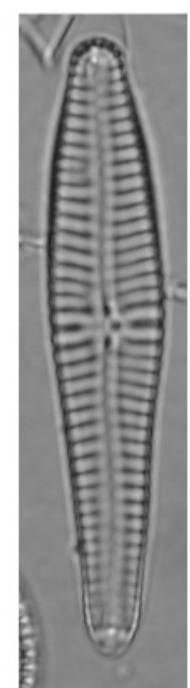

10

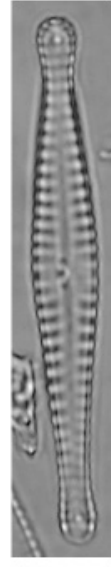

11
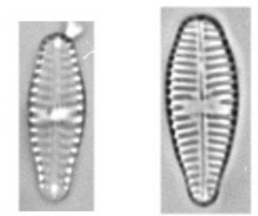

7

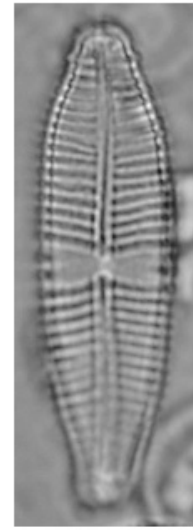

12

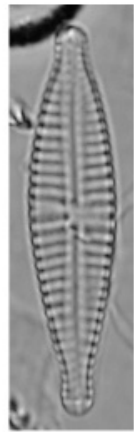

13

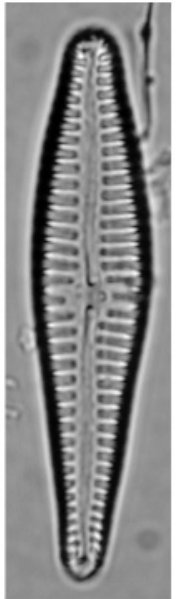

14

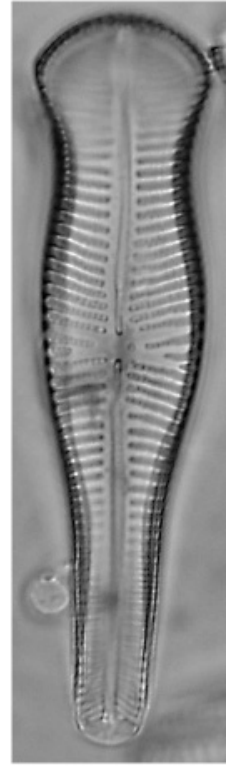

15

Explanation of Plate 9.

figs 1-3. Gomphonema coronatum Ehrenberg. figs 4, 5. Gomphonema subtile Ehrenberg. fig. 6. Gomphonema vibrio Ehrenberg. figs 7, 8. Gomphonema calcifugum Lange-Bertalot \& Reichardt. figs 9-11. Gomphonema clavatum Ehrenberg s. l. fig. 12. Gomphonema lapponicum (A. Cleve) Cleve-Euler. fig. 13. Gomphonema parvulum Kützing. fig. 14. Gomphonema sagitta Schumann. fig. 15. Gomphonema truncatum Ehrenberg. All LM micrographs (magnification $\times 1500)$. 
E. Witon \& A. Witkowski

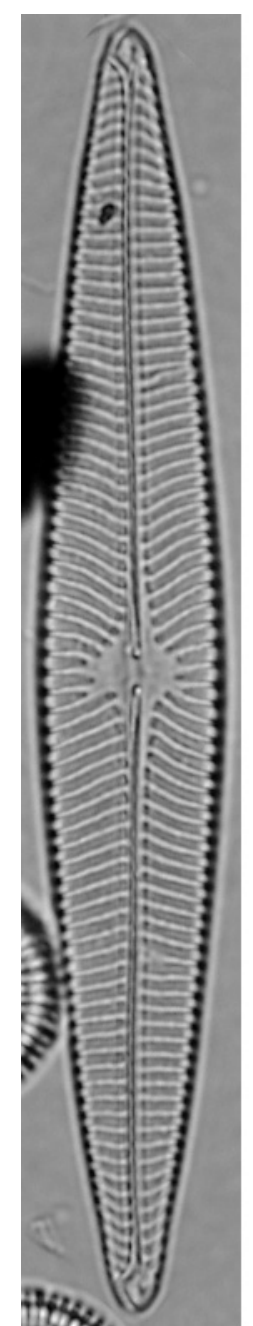

1

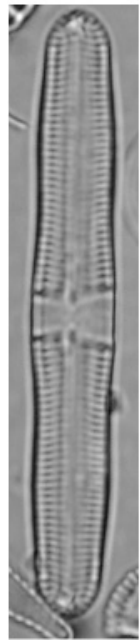

10

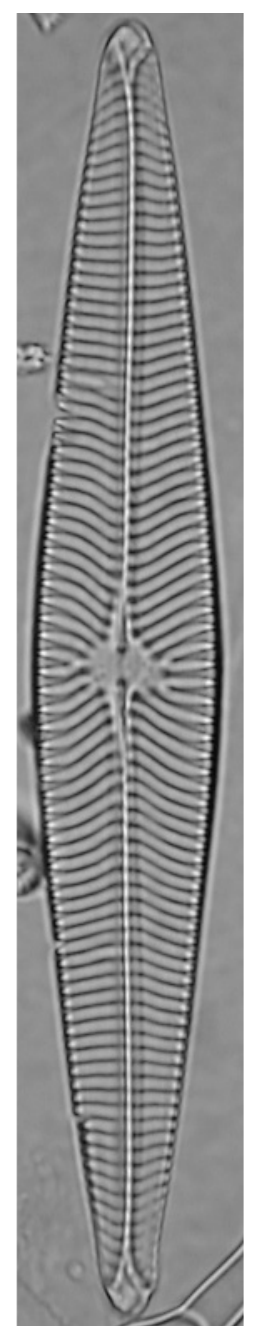

2

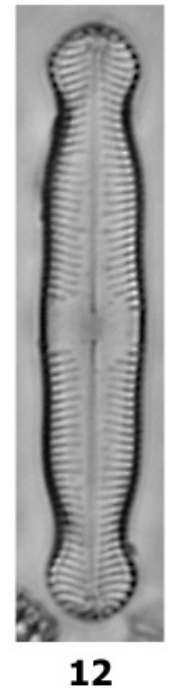

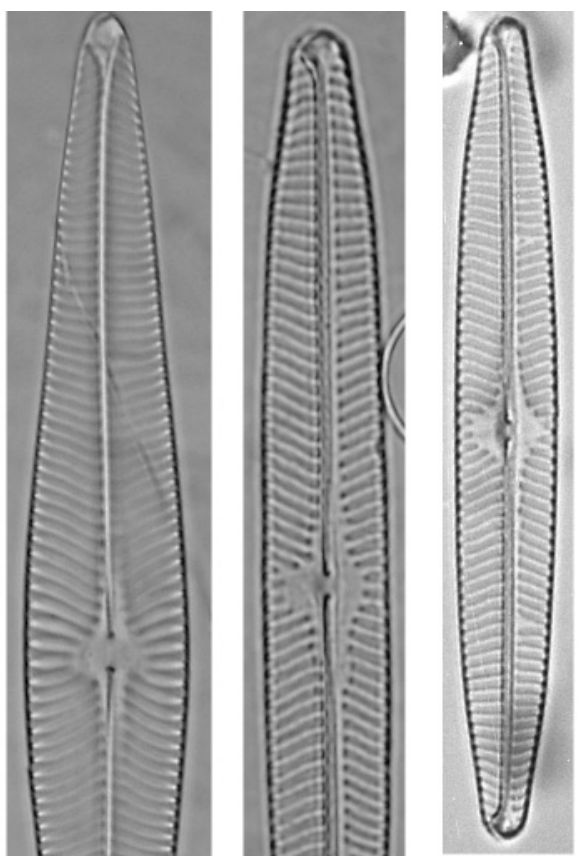
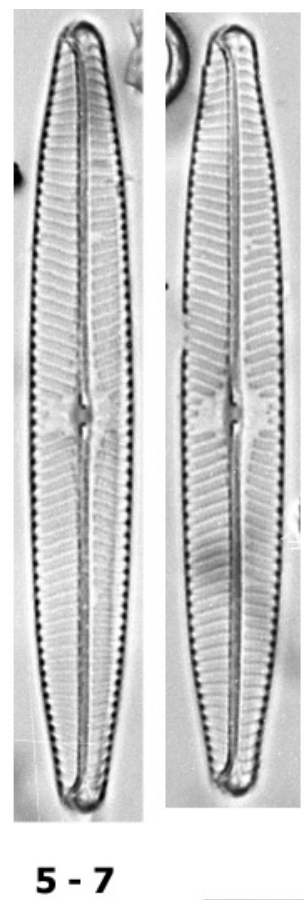

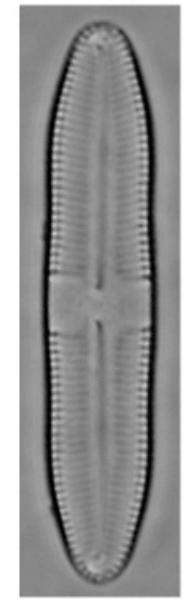

11
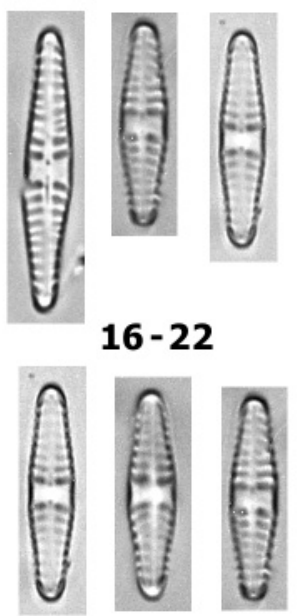

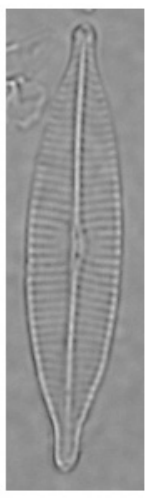

9

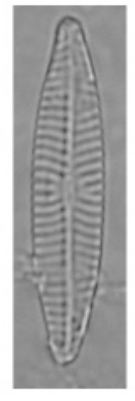

8

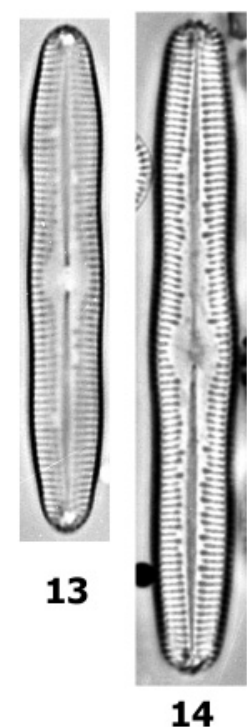

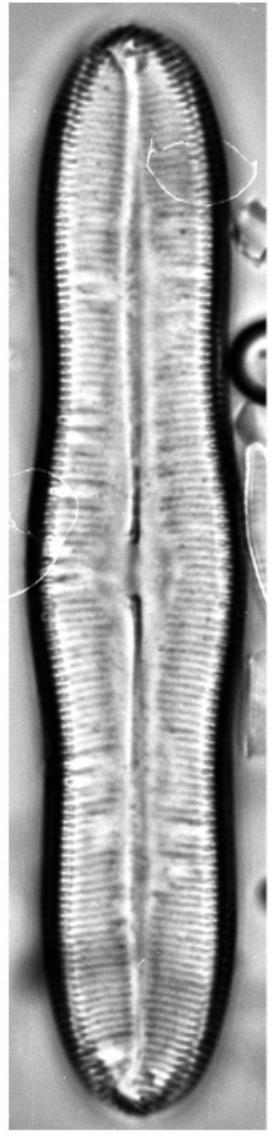

15

Explanation of Plate 10.

figs 1-3. Navicula radiosa Kützing. figs 4-7. Navicula angusta Grunow. fig. 8. Navicula veneta Kützing. fig. 9. Navicula gregaria Donkin s. l. figs 10, 11. Caloneis bacillum s. auct. fig. 12. Caloneis undulata (Gregory) Krammer. fig. 13. Caloneis tenuis (Gregory) Krammer. fig. 14. Caloneis pulchra Messikommer. fig. 15. Caloneis silicula (Ehrenberg) Cleve. figs 16-22. Hippodonta subcostulata (Hustedt) Lange-Bertalot, Metzeltin \& Witkowski. All LM micrographs (magnification $\times 1500$ ). 
Diatoms from Holocene freshwater sediments, Faeroe Islands

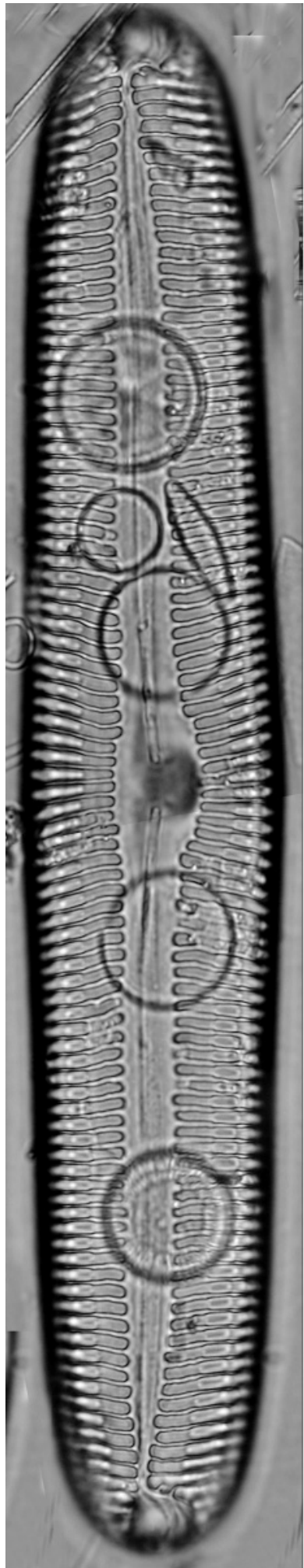

1

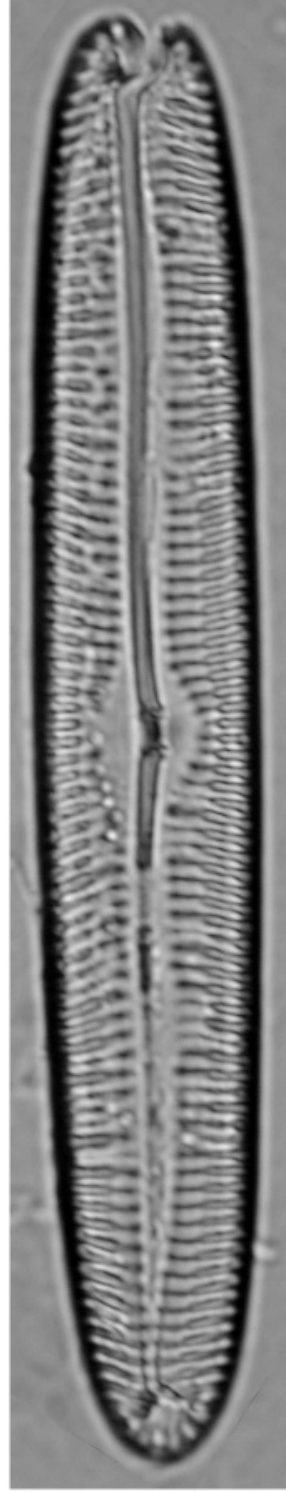

2

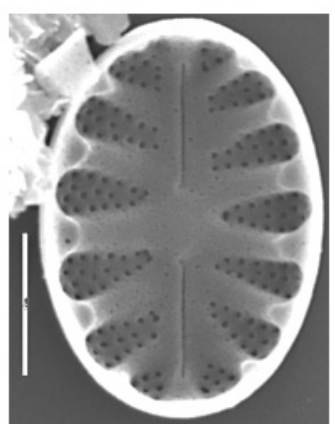

11

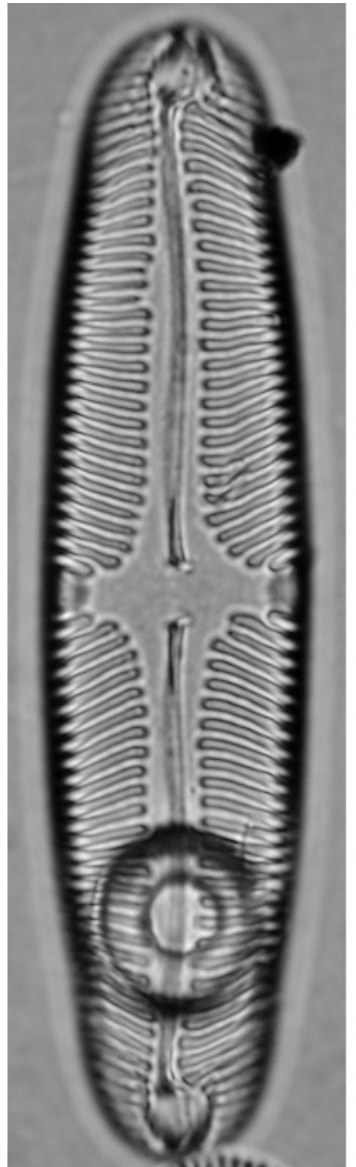

3
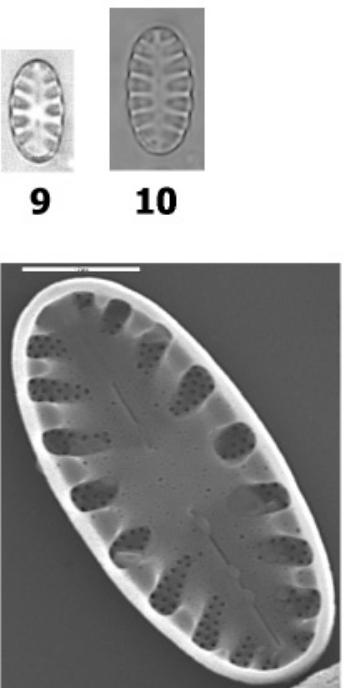

12
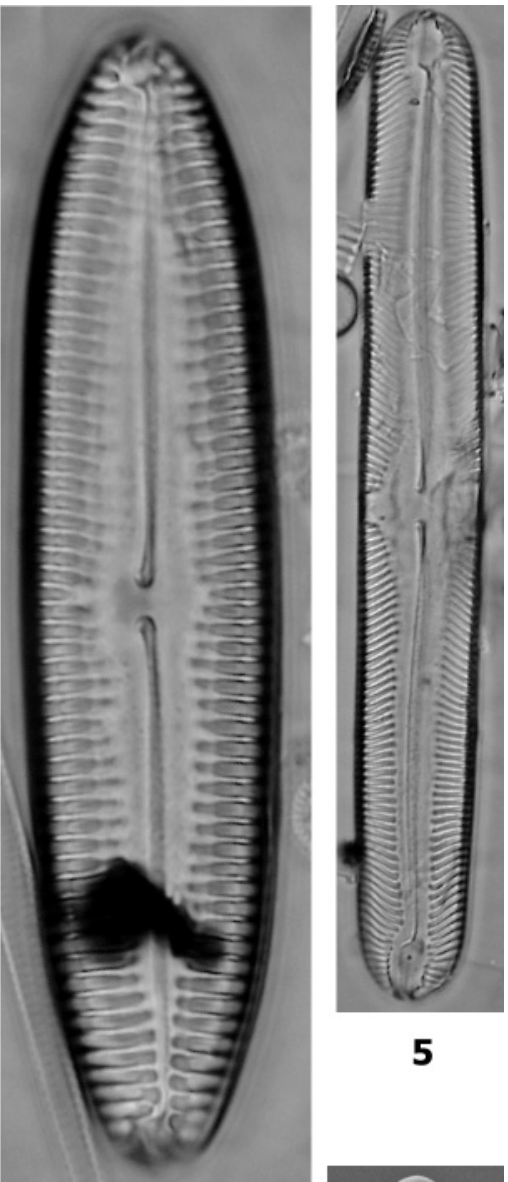

5

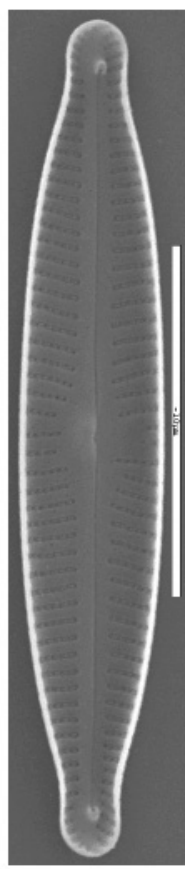

8

Explanation of Plate 11.

fig. 1. Pinnularia viridis (Nitzsch) Ehrenberg. fig. 2. Pinnularia viridiformis Krammer. fig. 3. Pinnularia divergens var. sublinearis Cleve, fig. 4. Pinnularia hemiptera (Kützing) Rabenhorst. fig. 5. Pinnularia stomatophora (Grunow) Cleve. fig. 6. Pinnularia subcapitata var. subrostrata Krammer. fig. 7. Pinnularia brandeli Cleve. fig. 8. Kobayasiella sp.. figs 9-12. Hygropetra balfouriana (Grunow ex. Cleve) Krammer \& Lange-Bertalot. figs 1-7, 9, 10 LM micrographs (magnification $\times 1500$ ); figs $8,11,12$ SEM micrographs (scale bars: $8=10 \mu \mathrm{m} ; 11,12=2 \mu \mathrm{m}$ ). 
E. Witon \& A. Witkowski

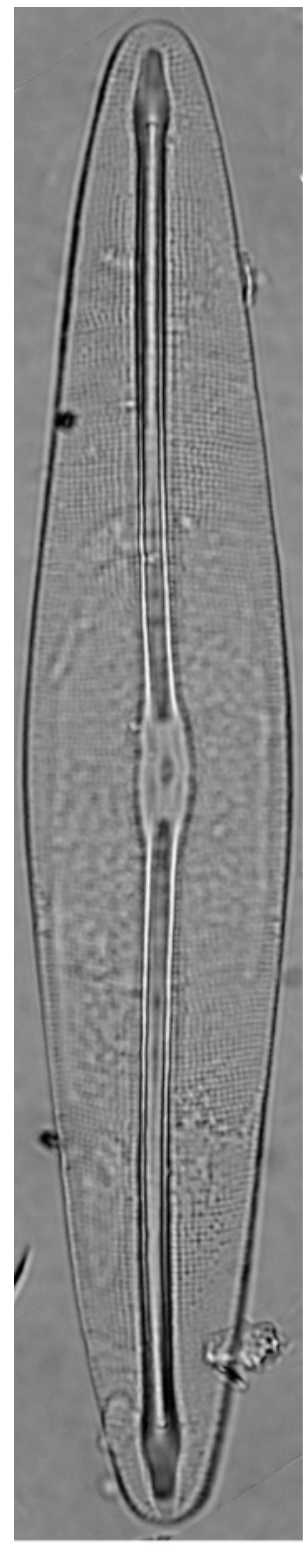

1

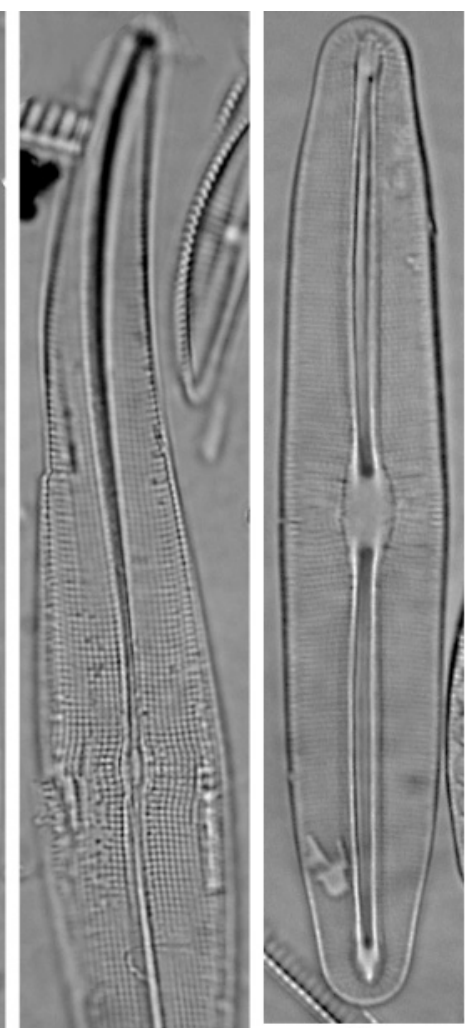

3
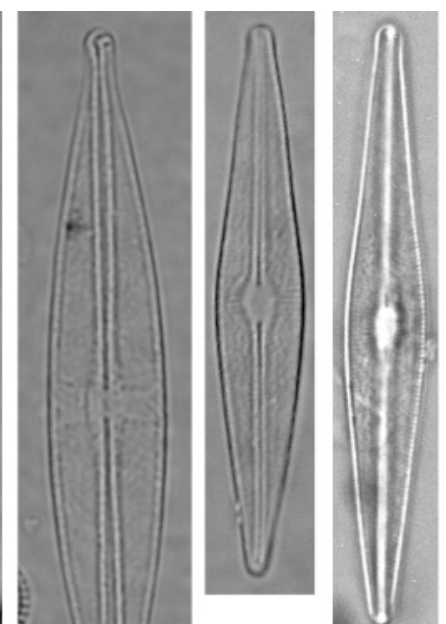

5 - 7

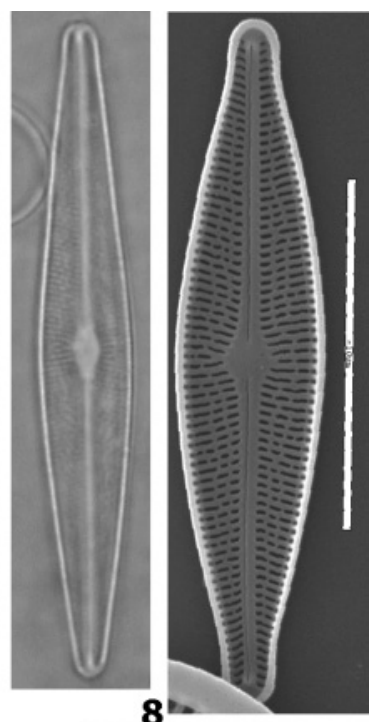

8

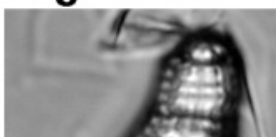

4

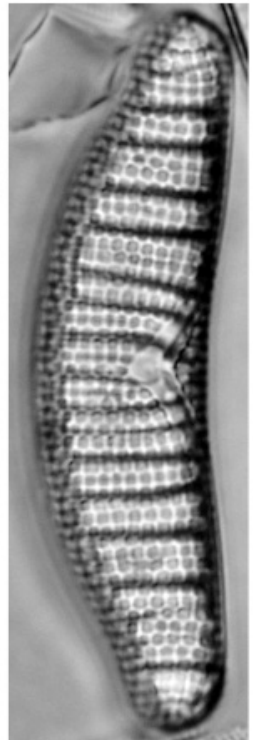

12

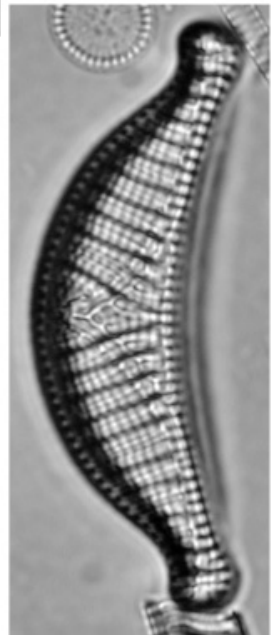

11

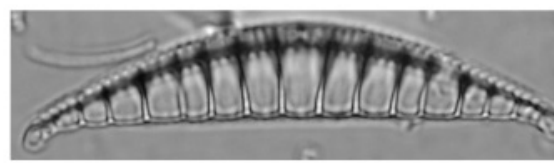

14

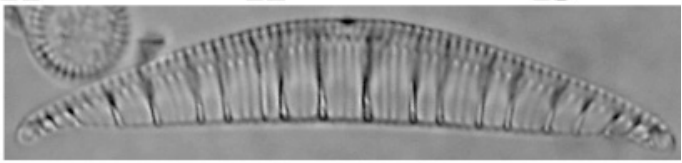

15

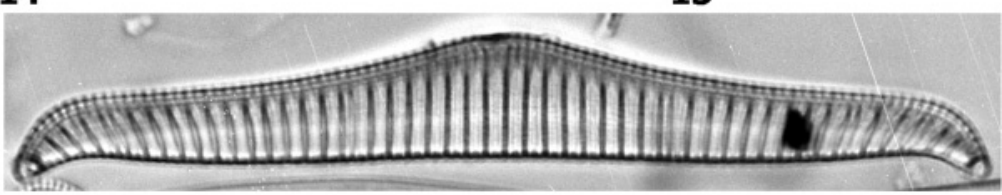

Explanation of Plate 12.

fig. 1. Frustulia erifuga Lange-Bertalot \& Krammer. fig. 2. Gyrosigama acuminatum (Kützing) Rabenhorst. fig. 3. Frustulia vulgaris (Thwaites) De Toni. fig. 4. Stauroneis neohyalina Lange-Bertalot. figs 5-8. Brachysira procera Lange-Bertalot. fig. 9. Brachysira brebissonii Ross. fig. 10. Brachysira zellensis (Grunow) Round \& Mann. fig. 11. Epithemia sorex Kützing. figs 12, 13. Epithemia adnata (Kützing) Brébisson. figs 14, 15. Rhopalodia rupestris (W. Smith) Krammer. fig. 16. Rhopalodia gibba (Ehrenberg) O. Müller var. gibba. figs 1-7, 9-16 LM micrographs (magnification $\times 1500$ ); fig. 8 SEM micrograph (scale bar: $10 \mu \mathrm{m}$ ). 
Diatoms from Holocene freshwater sediments, Faeroe Islands

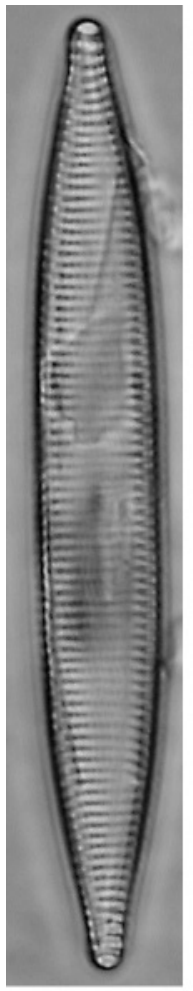

1

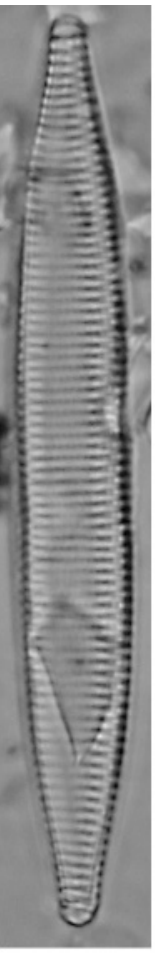

2

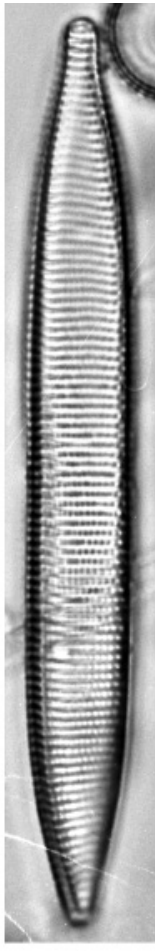

3

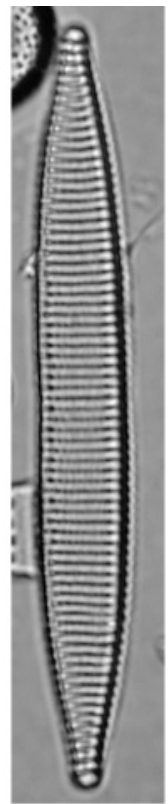

4

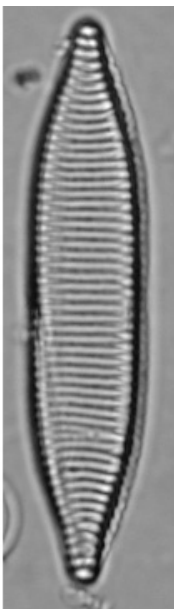

5
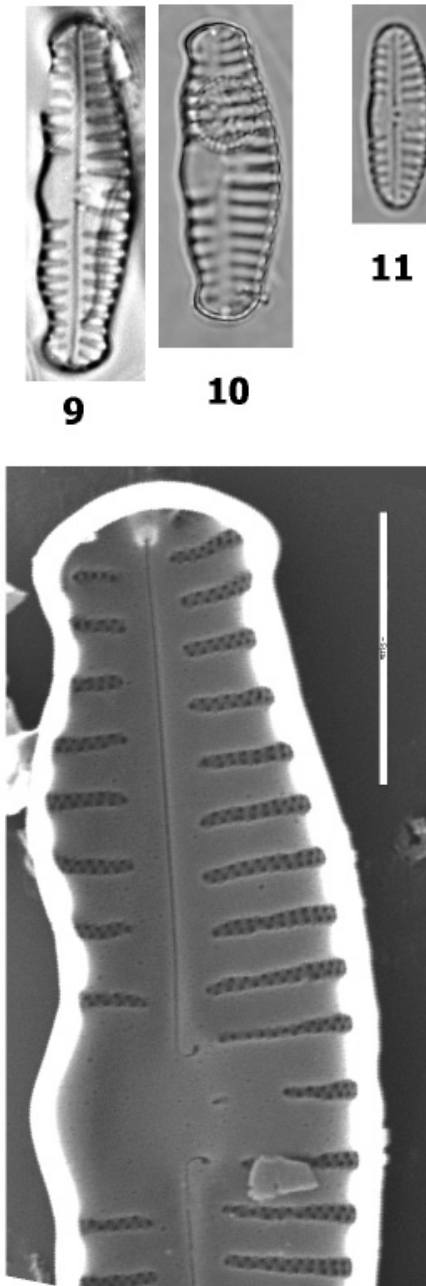

16

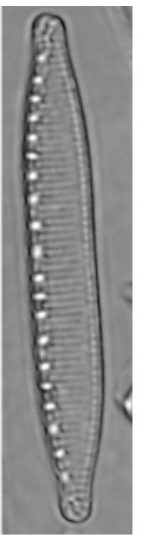

6

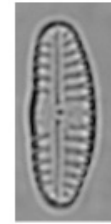

11
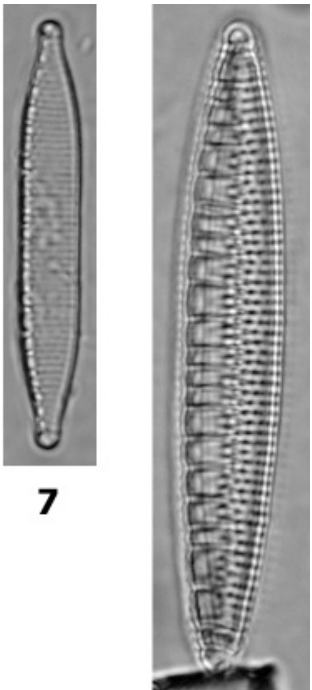

8

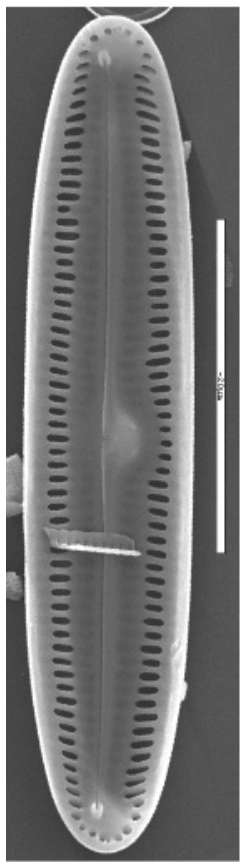

13

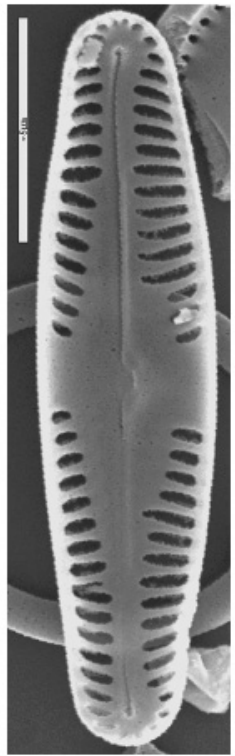

14

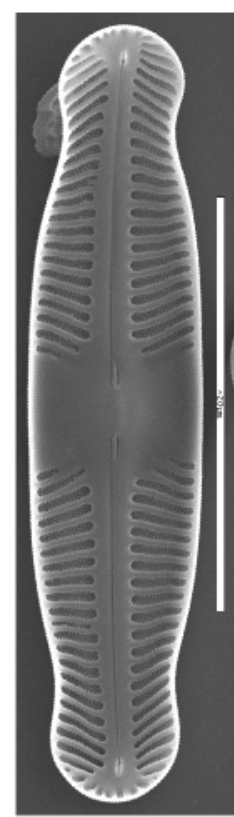

15

Explanation of Plate 13.

figs 1-5. Nitzschia angustata Grunow. fig. 6. Nitzschia hantzschiana Rabenhorst. fig. 7. Nitzschia cf. hantzschiana Rabenhorst. fig. 8. Nitzschia denticula Grunow. figs 9, 10. Reimeria uniseriata Sala et al. figs 11, 12. Reimeria sinuata (Gregory) Kociolek \& Stoermer. fig. 13. Caloneis sp. figs 14, 15. Pinnularia sp.. fig. 16. Reimeria sp.. fig. 17. Denticula tenuis Kützing. figs $1-12$ LM micrographs (magnification $\times 1500)$; figs $13-17$ SEM micrographs (scale bars: 13,15=20 $\mu \mathrm{m} ; 14,16,17=5 \mu \mathrm{m}$ ). 
Holmboe; Hannaea arcus (Ehrenberg) Patrick in Patrick \& Reimer

Lit.: Krammer \& Lange-Bertalot (1991a, p. 134, fig. 117: 8-13) Pl. 7, figs 26-28

Craticula cuspidata (Kützing) D.G. Mann

Syn.:Frustulia cuspidata Kützing; Navicula cuspidata (Kützing) Kützing; Navicula cuspidata var. heribaudii M. Pergallo in Héribaud

Lit.: Lange-Bertalot (2001, p. 111, fig. 82: 1-3, fig. 83: 1-2)

Pl. 6, fig. 7

Cyclostephanus invisitatus (Hohn \& Hellerman) Theriot,

Stoermer \& Håkansson

Syn.: Stephanodiscus invisitatus Hohn \& Hellerman; Stephanodiscus hantzschii var. striator Kalbe; Stephanodiscus incognitos Kuzmin \& Genkal

Lit.: Krammer \& Lange-Bertalot (1991a, p. 63, fig. 67: 3-4),

Håkansson (2002, p. 70, fig. 221-225)

Pl. 1, figs 12, 13; Pl. 2, fig. 4

Cyclotella antiqua W. Smith

Syn.: Cyclotella operculata var. antiqua Héribaud

Lit.: Krammer \& Lange-Bertalot (1991a, p. 48, fig. 47: 5-6, fig. 48: 1a-3)

Pl. 1, figs 14,15

\section{Cyclotella rossii Håkansson}

Syn.: Discoplea oligactis Ehrenberg; Cycotella oligactis (Ehrenberg) Ralphs in Pritchard; Cyclotella comta var. oligactis (Ehrenberg) Grunow in Van Heurck

Lit.: Krammer \& Lange-Bertalot (1991a, p. 60, fig. 64: 1-8)

Pl. 1, figs 1-5; P1. 2, figs 3, 4

Cymbella excisiformis Krammer

Lit.: Krammer (2002, p. 31, fig. 11: 1-23, fig. 12: 3-5, fig. 13: 1-8)

Cymbella helvetica Kützing

Syn.: Cymbella compacta Østrup

Lit.: Krammer \& Lange-Bertalot (1986, p. 324, fig. 132: 2-4, fig. 133: 1-8)

Pl. 4, figs 1, 2

Cymbella lange-bertalotii Krammer

Lit.: Krammer (2002, p. 152, fig. 179: 1-6)

P1. 4, figs 3-5

Cymbella aff. neocistula Krammer

Lit.: Krammer (2002, p. 94, fig. 85: 1-4, fig. 86: 1-7, fig. 87: 1-9, fig. 88: 1-8, fig. 89: 1-7, fig. 90: 1-8, fig. 91: 1-6)

So far this taxon has only been found with $3-5$ stigmata and this is the first report of a form with 6 .

Cymbella neoleptoceros var. tenuistriata Krammer

Lit.: Krammer (2002, p. 135, fig. 160: 1-6)

Pl. 4, figs 7,8

Cymbella pervarians Krammer

Lit.: Krammer (2002, p. 58, fig. 39: 8-18, fig. 41: 1-12, fig. 42: 1-12)

Pl. 4, fig. 9
Cymbella proxima Reimer

Syn.: Cocconema cistulum A. Schmidt; Cymbella cistula sensu Grunow in Van Heurck

Lit.: Krammer (2002, p. 106, fig. 92: 4-6, fig. 108: 1-6, fig. 109: 1-5, fig. 110: 1-3, fig. 111: 1-3)

Pl. 5, fig. 1

Cymbella cf. subtruncta Krammer

Lit.: Krammer (2002, p. 39, fig. 18: 16-21, fig. 19: 1-21)

Pl. 4, figs 10, 11

Cymbella vulgata Krammer

Lit.: Krammer (2002, p. 55, fig. 32: 7-13, fig. 36: 1-14, fig. 37: 16-21, fig. 38: 1-18, fig. 39: 1-7)

Pl. 4, fig. 6

Diadesmis biceps Arnott

Syn.: Navicula contenta Grunow; Navicula trinodis W. Smith f. minuta Grunow

Lit.: Krammer \& Lange-Bertalot (1986, p. 219, fig. 75: 1-5)

Pl. 8, figs 28, 29

Diadesmis perpusilla (Grunow) Lange-Bertalot

Syn.: Navicula prepusilla Grunow; Navicula gallica var. prepusilla (Grunow) Lange-Bertalot; Navicula flotowii Grunow

Lit.: Krammer \& Lange-Bertalot (1986, p. 220, fig. 75: 12-17) Pl. 8, figs $30-33$

Diatoma mesodon (Ehrenberg) Kützing

Syn.: Fragilaria mesodon Ehrenberg. Diatoma hiemalis var. mesodon (Ehrnberg) Grunow in Van Heurck

Lit.: Krammer \& Lange-Bertalot (1991a, p. 60, fig. 92: 1-4, fig. 99: 1-12)

Pl. 7, figs 7-9

Diatoma tenuis Agardh

Syn.: Diatoma tenuis var. elongatum Lyngbye; Diatoma elongatum (Lyngbye) Agardh; Diatoma mesoleptum Kützing Lit.: Krammer \& Lange-Bertalot (1991a, p. 97, fig. 96: 1-9) Pl. 7, fig. 6

Diatomella balfouriana Greville

Lit.: Krammer \& Lange-Bertalot (1986, p. 436, fig. 205: 4-8) Pl. 7, figs $13-19$

Didymosphenia geminata (Lyngbye) M. Schmidt

Syn.: Echinella geminata Lyngbye; Gomphonema geminatum (Lyngbye) Agardh

Lit.: Krammer \& Lange-Bertalot (1986, p. 381, fig. 166: 15)

Pl. 6, fig. 6

Encyonema neogracile Krammer

Syn.: (?) Cocconema gracile? Ehrenberg; (?)Cymbella gracilis Kützing; (?)Encyonema gracile Rabenhorst; Encyonema gracile var. Grunow in Van Heurck; Encyonema gracile f. minor Grunow in Van Heurck

Lit.: Krammer (1997, p. I/142, fig. 82: 1-13, fig. 83: 1-7, fig. 85: 1-12)

Pl. 5, figs 2, 3

Encyonema procerum Krammer

Lit.: Krammer (1997, p. I/95, fig. 32: 9-19)

Pl. 5, fig. 4 
Encyonema silesiacum (Bleisch) D.G. Mann

Syn.: Cymbella ventricosa Agardth; Cymbella silesiaca Bleisch in Rabenhorst; Cymbella minuta var. silesiaca (Bleisch) Reimer in Patrick \& Reimer

Lit.: Krammer (1997, p. I/72, fig. 4: 1-18, fig. 7: 6-19, fig. 9: 1-8, fig. 16: 1-11, fig. 17: 5-8)

Pl. 5, figs 2, 3

Eunotia arcus Ehrenberg

Syn.: Himantidium arcus Ehrenberg pro parte

Lit.: Krammer \& Lange-Bertalot (1991a, p. 184, fig. 147)

P1. 2, figs 1, 2

Eunotia circumborealis Nörpel-Schempp \& Lange-Bertalot Syn.: Eunotia septentrionalis var. bidens Hustedt sensu Simonsen; (?)Eunotia scandinavica f. angusta (Fontell) Cleve-Euler; Eunotia pectinalis var. undulata sensu Krasske Lit.: Krammer \& Lange-Bertalot (1991a, p. 197, fig. 143: 16-23)

Pl. 2, fig. 17

Eunotia implicata Nörpel-Schempp, Lange-Bertalot \& Alles Syn.: Eunotia impressa var. angusta Grunow in Van Heurck; Eunotia impressa var. angusta f. vix impressa Grunow in Van Heurck; Eunotia pectinalis var. minor f. impressa (Ehrenberg) Hustedt; Eunotia impressa Ehrenberg sensu Cleve-Euler; Eunotia impressa Ehrenberg; Himantidium minus Kützing; Himantidium pectinale var. minus (Kützing) Grunow; Eunotia pectinalis var. minor (Kützing) Rabenhorst sensu Grunow Lit.: Krammer \& Lange-Bertalot (1991a, p. 197, fig. 143: 1-9A) P1. 2, fig. 9

Eunotia media A. Cleve

Syn.: Eunotia parallela var. parallela Ehrenberg; Eunotia crassa Pantocsek \& Greguss; Eunotia pseudoparallela Cleve-Euler;

Eunotia parallela var. pseudoparallela Cleve-Euler

Lit.: Krammer \& Lange-Bertalot (1991a, p. 209, fig. 152: 4-7)

Pl. 2, fig. 18

Eunotia praerupta Ehrenberg

Syn.: Himantidium praeruptum Ehrenberg

Lit.: Krammer \& Lange-Bertalot (1991a, p. 186, fig. 148: 1-17, fig. 149: 1-7, fig. 150: 1-7)

Pl. 2, fig. 19

Fragilaria alpestris Krasske

Syn.: (?) Fragilaria capucina var. amphicephala (Kützing) Lange-Bertalot

Lit.: Krammer \& Lange-Bertalot (1991a, p. 141, fig. 111: 25-28)

Pl. 8, fig. 14

Fragilaria exigua Grunow in Cleve \& Moller

Syn.: Fragilaria virescens var. ? exigua Grunow in Van Heurck; Fragilaria exigua (W. Smith) Lemmermann; Triceratium

exiguum W. Smith; Fragilaria construens f. exigua (W. Smith) Hustedt

Lit.: Krammer \& Lange-Bertalot (1991a, p. 137, fig. 126: 11-18)

Pl. 8, figs $1-5,7$
Fragilaria construens var. binodis fo. borealis Foged

Lit.: Foged (1974, p. 56, fig. 3: 6), Krammer \& Lange-Bertalot (1991a, p. 164, fig. 130: 18, 19?)

Foged (1974) described $F$. construens v. binodis fo. borealis in a sample from an outflow of a small lake near Thingvellir in Iceland. He established a new forma based on faintly concave margins and coarsely punctate striae. Krammer \& LangeBertalot included this taxon with a question mark in Fragilaria robusta (Fusey) Manguin. The species, to a certain extent, resembles Fragilaria pseudoconstruens Marciniak, however, it differs with respect to valve shape, the sternum and the striation pattern.

Pl. 8, figs 8, 9

Fragilaria opacolineata Lange-Bertalot

Lit.: Lange-Bertalot \& Metzeltin (1996, p. 132, 340, fig. 7: 36-41B, fig. 111: 2-3)

Pl. 8, figs $15-17$

Fragilaria parasitica (W. Smith) Grunow Syn.: Synedra parasitica (W. Smith) Grunow in Van Heurck Lit.: Krammer \& Lange-Bertalot (1991a, p. 133, fig. 130: 1-8) Pl. 8, figs 18-20

Frustulia vulgaris (Thwaites) De Toni Syn.: Schizonema vulgare Thwaites Lit.: Krammer \& Lange-Bertalot (1986, p. 260, fig. 97: 1-6) P1. 12, fig. 3

Frustulia erifuga Lange-Bertalot \& Krammer

Syn.: Colletonema viridulum Brébisson ex Kützing; Schizonema viridulum (Brébisson) Rabenhorst; Vanheuricka viridula (Brébisson) Brébisson; Frustulia viridula (Brébisson) De Toni; Frustulia rhomboides var. viridula (Brébisson) Cleve; Frustulia rhomboides var. viridula f. hustedtii Germain Lit.: Lange-Bertalot (2001, p. 167, fig. 131: 9-10, fig. 132: 1-6, fig. 140: 1-2)

Pl. 12, fig. 1

Gomphomena acuminatum Ehrenberg var. acuminatum Syn.: Gomphonema brebissonii Kützing

Lit.: Krammer \& Lange-Bertalot (1986, p. 365, fig. 160: 1-12)

Gomphonema cf. affine Kützing

Syn.: Gomphonema lanceolatum sensu Hustedt (et al.) non Ehrenberg nec Agardh. (?)Gomphonema magnificum Gandhi

Lit.: Krammer \& Lange-Bertalot (1986, p. 366, fig. 161: 1-3)

Gomphonema calcifugum Lange-Bertalot \& Reichardt

Syn.: Gomphonema olivaceum var. minutissimum Hustedt

Lit.: Hustedt (1930, p. 378, fig. 720), Lange-Bertalot \& Genkal (1999, p. 53)

Pl. 9, figs 7,8

Gomphonema clavatum Ehrenberg

Syn.: Gomphonema longiceps Ehrenberg; Gomphonema mustela Ehrenberg; Gomphonema montanum Schumann; Gomphonema subclavatum Grunow; Gomphonema commutatum Grunow; Gomphonema (commutatum var.?) mexicanum Grunow; Gomphocymbella obliqua (Grunow) O. Müller Lit.: Krammer \& Lange-Bertalot (1986, p. 367, fig. 163: 1-12) Pl. 9, figs 9-11 
Gomphonema capitatum Ehrenberg

Syn.: Gomphonema trunctatum Ehrenberg; Gomphonema constrictum Ehrenberg; Gomphonema turgidum Ehrenberg

Lit.: Krammer \& Lange-Bertalot (1986, p. 369, fig. 159: 11-18)

Gomphonema coronatum Ehrenberg

Syn.: Gomphonema acuminatum var. coronatum (Ehrenberg) W. Smith

Lit.: Reichardt (1999, p. 43, fig. 49: 1-5, fig. 7-11, fig. 50: 1-8, fig. 51: 1-8)

Pl. 9, figs 1-3

Gomphonema lapponicum (A. Cleve) Cleve-Euler Syn.: Navicula petersenii f. gomphonemoides Hustedt Lit.: Reichardt (1999, p. 42, fig. 46: 1-10)

Pl. 9, fig. 12

Gomphonema sagitta Schumann

Syn.: Gomphonema subtille Ehrenberg; Gomphonema minusculum Krasske

Lit.: Krammer \& Lange-Bertalot (1986, p. 369, fig. 162: 10-13)

Pl. 9, fig. 14

Hippodonta subcostulata (Hustedt) Lange-Bertalot, Metzeltin \& Witkowski

Valves linear-lanceolate, with obtusely rounded apices, 14 $20 \mu \mathrm{m}$ long, 2.75-3.3 $\mu \mathrm{m}$ broad. Raphe straight with relatively distinct, somewhat expanded external central endings and straight terminal endings. Axial area very narrow, barely distinguishable, central area in a form of relatively broad fascia reaching the valve margins. Transapical striae relatively robust in the middle radiate, towards apices becoming convergent, $13-14$ in $10 \mu \mathrm{m}$. The valve outline and the shape of central area of this taxon resembles Hippodonta costulata (Grunow) Lange-Bertalot, Metzeltin \& Witkowski

Pl. 10, figs 16-22

Hygropetra balfouriana (Grunow ex Cleve) Krammer \& LangeBertalot

Bas.: Pinnularia balfouriana Grunow ex. Cleve

Lit.: Krammer (2000, p. 207, fig. 216: 1-9, fig. 15-19)

Pl. 11, figs 9-12

Navicula angusta Grunow

Syn.: Navicula cari var. angusta Grunow in Van Heurck; Navicula cincta var. angusta (Grunow) Cleve; Navicula cincta var. linearis Østrup; Navicula pseudocari Krasske; Navicula lobeliae Jørgensen

Lit.: Krammer \& Lange-Bertalot (1986, p. 97, fig. 28: 1-5)

P1. 10, figs 4-7

Navicula gregaria Donkin

Syn.: Navicula cryptcephala Kützing; Navicula gregalis Cholonky; Navicula gotlandica Grunow sensu Hustedt; Navicula phyllepta Kützing sensu Brockmann and sensu Hendey

Lit.: Krammer \& Lange-Bertalot (1986, p. 116, fig. 38: 10-15)

Pl. 10, fig. 9
Navicula veneta Kützing

Syn.: Navicula cryptocephala var. veneta (Kützing) Rabenhorst; Navicula cryptocephala var. subsalina Hustedt; Navicula lancettula Schumann

Lit.: Krammer \& Lange-Bertalot (1986, p. 104, fig. 32: 1-4) P1. 10, fig. 8

Neidium apiculatum Reimer

Lit.: Krammer \& Lange-Bertalot (1986, p. 250, fig. 100: 9)

Pl. 5, fig. 17

Nitzschia angustata (W.Smith) Grunow in Cleve \& Grunow Syn.: Tryblionella angustata W. Smith

Lit.: Krammer \& Lange-Bertalot (1997, p. 48, fig. 36: 1-5)

Pl. 13, figs $1-5$

Nitzschia denticula Grunow

Syn.: Denticula kuetzingii Grunow; Denticula obtusa W. Smith; Denticula inflata W. Smith; Denticula decipiens Arnott Lit.: Krammer \& Lange-Bertalot (1991a, p. 143, fig. 94: 3, 4, fig. 99: 11-23, fig. 100: 1-14, 18-22)

Pl. 13, fig. 8

Nitzschia hantzschiana Rabenhorst

Syn.: Nitzschia perpusilla Rabenhorst; Nitzschia frustulum var. glacialis Grunow in Van Heurck; Nitzschia frustulum $\mathrm{f}$. subserians Grunow in Van Heurck

Lit.: Krammer \& Lange-Bertalot (1997, p. 101, fig. 73: 9-18)

Pl. 13, figs 6, 7

Pinnularia brandeli Cleve

Lit.: Lange-Bertalot \& Metzeltin (1996, p. 206, fig. 44: 1-6) Pl. 11, fig. 7

Pinnularia divergens var. sublinearis Cleve

Syn.: Pinnularia divergens f. linearis Fontell; Pinnularia divergens var. fontellii Cleve-Euler; Pinnularia divergens var. elliptica sensu Krammer

Lit.: Krammer (2000, p. 62, fig. 30: 1-7, fig. 31: 1-8, fig. 32: 9) Pl. 11, fig. 3

Pinnularia hemiptera (Kützing) Rabenhorst

Syn.: Pinnularia acuminata W. Smith; Navicula instabilis A. Schmidt; Navicula hybrida Peragallo et Héribaud; Pinnularia debilis (Pantocsek) Cleve-Euler

Lit.: Krammer \& Lange-Bertalot (1986, p. 410, fig. 182: 1-3) Pl. 11, fig. 4

Pinnularia neomajor Krammer

Syn.: Navicula major ex. rec Grunow in A. Schmidt; (?)Frustulia major Kützing; Navicula major Kützing; Pinnularia major sensu Cleve

Lit.: Krammer (2000, p. 165, fig. 6: 1-4, fig. 62: 1-5, fig. 63: 1)

Pinnularia ovata Krammer

Syn.: Navicula divergens var. elliptica Grunow; Pinnularia divergens var. elliptica (Grunow) Cleve; Pinnularia episcopalis sensu Hustedt

Lit.: Krammer (2000, p. 64, fig. 35: 5-8, fig. 36: 1-5, fig. 37:1-4)

Pinnularia platycephala (Ehrenberg) Cleve

Bas.: Stauroptera platycephala Ehrenberg

Syn.: Pinnularia platystoma Hustedt 
Diatoms from Holocene freshwater sediments, Faeroe Islands

Lit.: Krammer (2000, p. 68, fig. 2: 1, fig. 39: 6, fig. 44: 1-7)

Pinnularia rupestris Hantzsch in Rabenhorst

Syn.: Pinnularia viridis var. rupestris (Hantzsch) Cleve; Pinnularia reinschiana A. Mayer

Lit.: Krammer (2000, p. 135, fig. 118: 1-12)

Pinnularia stomatophora (Grunow) Cleve

Syn.: Navicula stomatophora Grunow; Pinnularia stomatophora var. triundulata Fontell; Pinnularia substomatophora Hustedt; Pinnularia stomatophoroides A. Mayer

Lit.: Krammer \& Lange-Bertalot (1986, p. 406, fig. 178: 8-10, fig. 179: 1, fig. 18: 5)

Pl. 11, fig. 5

Pinnularia subcapitata var. subrostrata Krammer

Lit.: Krammer (2000, p. 118, fig. 38: 12-18, fig. 90: 18-23)

Pl. 11, fig. 6

Pinnularia turbulenta (Cleve-Euler) Krammer

Bas.: Pinnularia mesolepta var. turbulenta Cleve-Euler

Lit.: Krammer (2000, p. 100, fig. 83: 1-6)

Placoneis cf. clementis Grunow

Bas.: Navicula clementis Grunow

Syn.: Navicula exigua (Gregory) Grunow; Navicula clementis var. rhombica Brockmann; Navicula inclementis Hendey

Lit.: Krammer \& Lange-Bertalot (1986, p. 139, fig. 47: 1-9, fig. 53: 3)

Pl. 6, fig. 11

Reimeria sinuata (Gregory) Kociolek \& Stoermer

Lit.: Sala et al. (1993, p. 442-443, fig. 2-10)

Pl. 13, figs 11, 12

Reimeria uniseriata (Gregory) Sala, Guerrero \& Ferrario Syn.: Cymbella sinuata Gregory sensu Schumann \& Archibald Lit.: Sala et al. (1993, p. 445)

Pl. 13, figs 9, 10

Rhopalodia gibba (Ehrenberg) O. Müller var. gibba

Syn.: Navicula gibba Ehrenberg; Epithemia gibba (Ehrenberg)

Kützing; Epithemia ventricosa Kützing; Rhopalodia ventricosa

(Kützing) O. Müller; Rhopalodia gibba var. ventricosa (Kützing) Pergallo

Lit.: Krammer \& Lange-Bertalot (1997, p. 159, fig. 110: 1, fig. 111: 1-13)

Pl. 12, fig. 16

Rhopalodia rupestris (W. Smith) Krammer Syn.: Epithemia rupestris W. Smith; Rhopalodia gibberula var. rupestris (W. Smith) O. Müller

Lit.: Krammer \& Lange-Bertalot (1997, p. 165, fig. 115: 1-8) Pl. 12, figs 14, 15

Stauroneis neohyalina Lange-Bertalot \& Krammer

Syn.: Stauroneis anceps var. siberica Grunow in Cleve \& Grunow

Lit.: Lange-Bertalot \& Metzeltin (1996, p. 104, fig. 35: 7-10)

Pl. 12, fig. 4

Staurosira construens Ehrenberg

Syn.: Fragilaria construens (Ehrenberg) Grunow
Lit.: Krammer \& Lange-Bertalot (1991a, p. 153, fig. 132: 1-34, fig. 129: 21-27, fig. 131: 5-6), Krammer \& Lange-Bertalot (2000, p. 584)

Pl. 8, fig. 21

Staurosira martyi (Héribaud) Lange-Bertalot

Bas.: Opephora martyi Héribaud

Syn.: Fragilaria martyi (Héribaud) Lange-Bertalot; Martyana martyi (Héribaud) Round in Round et al. (1990)

Lit.: Krammer \& Lange-Bertalot (1991a, p. 160, fig. 133: 28-31), Witkowski et al. (1996). Krammer \& Lange-Bertalot (2000, p. 586)

Pl. 8, fig. 6

Staurosira pseudoconstruens (Marciniak) Lange-Bertalot

Bas.: Fragilaria pseudoconstruens Marciniak

P1. 8, figs 22-24

Lit.: Krammer \& Lange-Bertalot (1991a, p. 163, fig. 130: 25-30), Krammer \& Lange-Bertalot (2000, p. 587)

Surirella amphioxys W. Smith

Syn.: Surirella moelleriana Grunow ex Moller; Surirella moelleriana sensu Germain

Lit.: Krammer \& Lange-Bertalot (1986, p. 189, fig. 138: 1-5, fig. 39: 1-8)

Pl. 6, fig. 12

Tabellaria flocculosa sensu lato (Roth) Kützing

Syn.: Diatoma fenestratum Lyngbye

Lit.: Krammer \& Lange-Bertalot (1991, p. 106, fig. 105: 1-4, fig. 107: 8)

Pl. 7, figs 20-23

Tabellaria flocculosa sensu stricto (Roth) Kützing

Syn.: Conferva flocculosa Roth

Lit.: Krammer \& Lange-Bertalot (1997, p. 108, fig. 106: 1-3, fig. 107: 7, 11, 12)

Pl. 7, figs 24, 25

\section{APPENDIX B: SPECIES LIST}

\section{Freshwater species}

Achnanthes daonensis Lange-Bertalot

Achnanthes didyma Hustedt

Achnanthes holstii Cleve

Achnanthes lanceolata (Brébisson) Grunow

Achnanthes laterostrata Hustedt

Achnanthes pusilla (Grunow) de Toni

Amphora copulata (Kützing) Schoeman

Amphora inariensis Krammer

Amphora veneta Kützing

Aneumastus rostratus (Hustedt) Lange-Bertalot

Aulacoseira distans (Ehrenberg) Simonsen

Aulacoseira italica (Ehrenberg) Simonsen

Aulacoseira subarctica (O. Müller) Haworth

Aulacoseira valida (Grunow) Krammer

Brachysira brebissonii Ross

Brachysira procea Lange-Bertalot \& Moser

Brachysira zellensis (Grunow) Round \& D.G. Mann

Caloneis cf. bacillum (Grunow) Cleve

Caloneis pulchra Messikommer

Caloneis silicula (Ehrenberg) Cleve 
Caloneis tenuis (Gregory) Krammer

Caloneis undulata (Gregory) Krammer

Cavinula cocconeiformis (Gregory) D.G. Mann \& Stickle

Ceratoneis arcus (Ehrenberg) Kützing var. arcus

Cocconeis placentula var. lineata (Ehrenberg) Van Heurck

Craticula cuspidata (Kützing) D.G. Mann

Cyclostephanos invisitatus (Hohn \& Hellerman) Theriot,

Stoermer \& Håkansson

Cyclotella antiqua $\mathrm{W}$. Smith

Cyclotella ocellata Pantocsek

Cyclotella radiosa (Grunow) Lemmermann

Cyclotella rossii Håkansson

Cymbella aff. neocistula Krammer

Cymbella cf. subtruncta Krammer

Cymbella excisiformis Krammer

Cymbella helvetica Kützing

Cymbella lange-bertalotii Krammer

Cymbella neoleptoceros var. tenuistriata Krammer

Cymbella pervarians Krammer

Cymbella proxima Reimer

Cymbella vulgata Krammer

Denticula tenuis Kützing

Diadesmis biceps Arnott

Diadesmis perpusilla (Grunow) Lange-Bertalot

Diatoma mesodon (Ehrenberg) Kützing

Diatoma tenuis Agardh

Diatomella balfouriana Greville

Didymosphenia geminata (Lyngbye) M. Schmidt

Diploneis petersenii Hustedt

Diploneis pseudovalis (Hilse) Cleve

Encyonema neogracile Krammer

Encyonema procerum Krammer

Encyonema silesiacum (Bleisch) D.G. Mann

Epithemia adnata (Kützing) Brébisson

Epithemia sorex Kützing

Epithemia turgida (Ehrenberg) Kützing

Eucocconeis alpestris (Brun) Lange-Bertalot

Eucocconeis flexella (Kützing) Cleve var. flexella

Eucocconeis laevis (Østrup) Lange-Bertalot

Eunotia arcus Ehrenberg

Eunotia circumborealis Nörpel-Schempp \& Lange-Bertalot

Eunotia implicata Nörpel-Schempp, Lange-Bertalot \& Alles

Eunotia media Cleve

Eunotia praerupta Ehrenberg

Eunotia pseudopectinalis (Brébisson) Kützing

Fragilaria alpestris Krasske

Fragilaria capucina var. distans (Grunow) Lange-Bertalot

Fragilaria capucina var. perminuta (Grunow) Lange-Bertalot

Fragilaria capucina var. vaucheriae (Kützing) Lange-Bertalot

Fragilaria construens var. binodis fo. borealis Foged

Fragilaria exigua Grunow in Cleve \& Moeller

Fragilaria opacolineata Lange-Bertalot

Frustulia erifuga Lange-Bertalot \& Krammer

Frustulia vulgaris (Thwaites) De Toni

Gomphomena acuminatum Ehrenberg var. acuminatum

Gomphonema calcifugum Lange-Bertalot \& Reichardt

Gomphonema capitatum Ehrenberg

Gomphonema cf. affine Kützing

Gomphonema clavatum Ehrenberg
Gomphonema coronatum Ehrenberg

Gomphonema lapponicum (Cleve) Cleve-Euler

Gomphonema parvulum Kützing

Gomphonema sagitta Schumann

Gomphonema truncatum Ehrenberg

Gomphonema vibrio Ehrenberg

Gomphonemma subtile Ehrenberg

Gyrosigma acuminatum (Kützing) Rabenhorst

Hippodonta subcostulata (Hustedt) Lange-Bertalot, Metzeltin \&

Witkowski

Hygropetra balfouriana (Grunow ex Cleve) Krammer \& Lange-

Bertalot

Meridion circulae (Greville) Agardh

Navicula angusta Grunow

Navicula gegaria Donkin

Navicula radiosa Kützing

Navicula schmasmannii Hustedt

Navicula veneta Kützing

Neidium apiculatum Reimer

Nitzschia angustata (W. Smith) Grunow in Cleve \& Grunow

Nitzschia denticula Grunow

Nitzschia hantzschiana Rabenhorst

Pinnularia brandeli Cleve

Pinnularia cf. viridis (Nitzsch) Ehrenberg

Pinnularia divergens var. sublinearis Cleve

Pinnularia hemiptera (Kützing) Rabenhorst

Pinnularia neomajor Krammer

Pinnularia ovata Krammer

Pinnularia platycephala (Ehrenberg) Cleve

Pinnularia rupestris Hantzsch in Rabenhorst

Pinnularia stomatophora Grunow

Pinnularia subcapitata var. subrostrata Krammer

Pinnularia turbulenta (Cleve-Euler) Krammer

Pinnularia viridiformis Krammer

Placoneis cf. clementis Grunow

Reimeria sinuata (Gregory) Kociolek \& Stoermer

Reimeria uniseriata (Gregory) Kociolek \& Stoermer

Rhopalodia rupestris (W. Smith) Krammer

Stauroneis neohyalina Lange-Bertalot \& Krammer

Staurosira construens Ehrenberg

Staurosira martyi (Héribaud) Lange-Bertalot

Staurosira parasitica (W. Smith) Grunow

Staurosira pseudoconstruens (Marciniak) Lange-Bertalot

Surirella amphioxys W. Smith

Tabelaria flocculosa (Roth) Kützing

\section{Brackish-water species}

Achnanthes brevipes Agardh var. brevipes

Campylodiscus clypeus Ehrenberg

Cocconeis scutellum Ehrenberg var. scutellum

Cocconeis speciosa Gregory

Diploneis litoralis (Donkin) Cleve var. litoralis

Diploneis smithii (Brébisson) Cleve var. smithii

Diploneis stroemii Hustedt

Fallacia forcipata (De Toni) Stickle \& D.G. Mann

Grammatophora oceanica (Ehrenberg 1854 pro parte) Grunow

Nitzschia coarctata Grunow

Pleurosigma normanii Ralfs

Rhabdonema arcuatum (? Agardh) Kützing var. arcuatum 
Rhabdonema minutum Kützing

Rhopalodia gibba (Ehrenberg) O. Müller var. gibba

Synedra tabulata (Agardh) Kützing var. tabulata

Thalassiosira eccentrica (Ehrenberg) Cleve

\section{Marine species}

Actinoptychus senarius (Ehrenberg) Ehrenberg

Amphora marina W. Smith

Bacterosira batyomphala (Cleve) Syvertsen \& Hasle

Caloneis undulata (Gregory) Krammer

Cocconeis costata Gregory var. costata

Cocconeis gutatta Hustedt

Cocconeis pinnata Gregory ex Greville

Cocconeis pseudomarginata Gregory

Dimeregramma fulvum (Gregory) Ralfs in Pritchard

Diploneis bombus Ehrenberg

Diploneis notabilis (Greville) Cleve

Diploneis vacillans (A. Schmidt) Cleve var. vacillans

Grammatophora angulosa var. islandica (Ehrenberg) Grunow

Grammatophora marina (Lyngbye) Kützing

Lyrella lyra (Ehrenberg) Karayeva

Navicula distans (W. Smith) A. Schmidt

Navicula normalis Hustedt

Odontella aurita (Lyngbye) Agardh

Opephora marina (Gregory) Petit

Paralia sulcata (Ehrenberg) Cleve

Plagiogramma staurophorum (Gregory) Heiberg

Rhizosolenia hebetata Bailey

Rhoicosphenia marina (W. Smith) M. Schmidt

Rhopalodia acuminata Krammer

Thalasionema nitzschioides Grunow

Thalassiosira decipiens (Grunow) Jørgensen

Thalassiosira nordenskioeldii Cleve

Trachyneis aspera (Ehrenberg) Cleve

\section{Manuscript received 10 January 2003 \\ Manuscript accepted 17 July 2003}

\section{REFERENCES}

Bennike, O., Böcher, J., Konradi, P., Kuijpers, A. \& Larsen, B. 1998. Macrofossil studies of lacustrine sediments from Skálafjørður, the Faroe Islands: preliminary results. Fróðskaparrit, 46: 267-275.

Boldreel, L.O. \& Andersen, M.S. 1995. The relationship between the distribution of Tertiary sediments, tectonic processes and deep water circulation around the Faeroe Islands. In: Scrutton, R.A., Stoker, M.S., Shimmield, G.B. \& Tudhope, A.W. (Eds), The Tectonics, Sedimentation and Paleoceanography of the North Atlantic region. Geological Society, London, Special Publications, 90: 145-158.

Bondevik, S., Svendsen, J.I., Johnsen, G., Mangerud, J. \& Kaland, P.E. 1997. The Storegga tsunami along the Norwegian coast, its age and runup. Boreas, 26: 29-53.

Brun, J. 1901. Diatomees d'eau douce de l'ile Jan Mayen et de la cote du Groenland, recoltees par l'expdition suedoise de 1899. Kungliga Vetenskaps Svenska Akademiens Handlingar, 26: 1-22.

Cleve, P.T. 1873. On diatoms from the Arctic Sea. Kungliga Vetenskaps Svenska Akademiens Handlingar, 1: 1-28.

Cleve, P.T. 1896. Planktonundersökningar: Vegetabilisk Plankton. Kungliga Vetenskaps Svenska Akademiens Handlingar, 22/5: 1-31.

Cleve, P.T. 1898. The plankton of the North Sea, the English Channel and the Skagerak in 1898. Kungliga Vetenskaps Svenska Akademiens Handlingar, 32: 1-53.
Cleve, P.T. 1900. Notes on some Atlantic plankton organisms. Kungliga Vetenskaps Svenska Akademiens Handlingar, 34: 20.

Cleve, P.T. \& Grunow, A. 1880. Beiträge zur Kenntnis der arktischen Diatomeen. Kungliga Vetenskaps Svenska Akademiens Handlingar, 17/2: $1-121$.

Cleve-Euler, A. 1951-1955. Die Diatomeen von Schweden und Finnland. Kungliga Vetenskaps Svenska Akademiens Handlingar, Series 4: 2/1: 1-163 (1961). 3/3: 1-153 (1952). 4/1: 1-158 (1953). 4/5: 1-225 (1953). 5/4: 1-232 (1955).

Dawson, A.G., Long, D. \& Smith, D.E. 1988. The Storegga slides: evidence from eastern Scotland for a possible tsunami. Marine Geology, 82: 271-276.

Dawson, S. \& Smith, D.E. 2000. The sedimentology of Middle Holocene tsunami facies in northern Sutherland, Scotland, UK. Marine Geology, 170: 69-79.

Denys, L. 1992. A check-list of the diatoms in the Holocene deposits of the western Belgian coastal plain with a survey of their apparent ecological requirements. I. Introduction, ecological code and complete list. Professional Paper Belgian Geological Survey, 246: 1-411.

Foged, N. 1964. Freshwater Diatoms from Spitsbergen. Tromse Museums Skrifter Vol XI. Universitetforlaget, Tromsø/Oslo, 205 pp.

Foged, N. 1974. Freshwater diatoms in Iceland. Bibliotheca Phycologica, 15: $1-192$.

Grauert, M., Björck, S. \& Bondevik, S. 2001. Storegga tsunami deposits in a coastal lake on Suðuroy, the Faroe Islands. Boreas, 30: 263-271.

Håkansson, H. 1990. A comparison of Cyclotella krammeri sp. nov. and C. schumannii Håkansson stat. nov. with similar species. Diatom Research, 5: 261-271.

Håkansson, H. 2002. A compilation and evaluation of species in the general Stephanodiscus, Cyclostephanos and Cyclotella with a new genus in the family Stepahnodiscaceae. Diatom Research, 17: 1-139.

Håkansson, H. \& Ross, R. 1984. Proposals to designate conserved types for Cymbella and Cyclotella, and to conserve Rhopalodia against Pyxidicula (all Bacillariophyceae). Taxon, 33: 525-531.

Hoffman, G. 1994. Aufwuchs-Diatomeen in See und ihre Eidnung als Indikatoren der Trophie. Bibliotheca Diatomologica, 30. J. Cramer, Stuttgart, 1-393.

Hustedt, F. 1930. Bacillariophyta. In: Pascher, A. (Ed.), Süßwasserflora von Mitteleuropa, 10. Gustar Fischer, Jena, Germany, 1-466.

Hustedt, F. 1937. Diatomeen von Island, Spitzbergen und Faröer-Inseln. Botanisches Archiv, 38: 152-207.

Jiang, H., Seidenkrantz, M.S., Knudsen, K.L. \& Eriksson, J. 2001. Diatom surface sediment assemblages around Iceland and their relationships to oceanic environmental variables. Marine Micropaleontology, 41: 73-96.

Jørgensen, G. \& Rasmussen, J. 1986. Glacial striae, roches moutonnées and ice movements in the Faeroe Islands. Geological Survey of Denmark Report, Series, C7: 1-114.

Jozkow, J. 2000. Environmental changes in Late Glacial and Holocene in the area of the Faeroe Islands based on the diatom analyses. MSc thesis. University of Szczecin, Poland.

Juul, M. 1992. Den Holocane udvikling i Skálafjord, Farøerne. Student thesis. Institute of Geology, Aarhus University, $92 \mathrm{pp}$.

Koc, N. \& Jansen, E. 1992. A high-resolution diatom record of the last deglaciation from the SE Norwegian Sea: Documentation of rapid climatic changes. Paleooceanography, 7(4): 499-520.

Koc, N. \& Schrader, H. 1990. Surface sediment diatom distribution and Holocene paleotemperature variations in the Greenland, Iceland and Norwegian Sea. Paleooceanography, 5(4): 557-580.

Kohly, A. 1998. Diatom flux and species composition in the Greenland Sea and the Norwegian Sea in 1991-1992. Marine Geology, 145: 293-312.

Krammer, K. 1992. Pinnularia eine Monographie der europäischen Taxa. Bibliotheca Diatomologica, 26. J. Cramer, Stuttgart, 1-353.

Krammer, K. 1997. Die cymbelloiden Diatomeen. Eine Monographie der weltweit bekannten Taxa. Allgemeines und Encyonema Part. Teil 1. Bibliotheca Diatomologica, 36. J. Cramer, Stuttgart, 1-469.

Krammer, K. 2000. The genus Pinnularia. In: Lange-Bertalot, H. (Ed.), Diatoms of Europe, 1. A.R.G. Gantner, Ruggell, Germany, 1-703.

Krammer, K. 2002. Cymbella. In: Lange-Bertalot, H. (Ed.), Diatoms of Europe, 3. A.R.G. Gantner, Ruggell, Germany, 1-584. 
Krammer, K. \& Lange-Bertalot, H. 1986. Bacillariophyceae. Teil 1, Naviculaceae. In: Ettl, H., Gerloff, J., Heynig, H. \& Mollenhauer, D. (Eds), Süßwasserflora von Mitteleuropa, 2/1. Gustav Fischer, Stuttgart, $876 \mathrm{pp}$

Krammer, K. \& Lange-Bertalot, H. 1991a. Bacillariophyceae. Teil 3, Centrales, Fragilariaceae, Eunotiaceae. In: Ettl, H., Gerloff, J., Heynig, H. \& Mollenhauer, D. (Eds), Süßwasserflora von Mitteleuropa, 2/3. Gustav Fischer, Stuttgart, 576 pp.

Krammer, K. \& Lange-Bertalot, H. 1991b. Bacillariophyceae. Teil 4, Achnanthaceae. In: Ettl, H., Gärtner, G., Gerloff, J., Heynig, H. \& Mollenhauer, D. (Eds), Süßwasserflora von Mitteleuropa, 2/4. Gustav Fischer, Stuttgart, 473 pp.

Krammer, K. \& Lange-Bertalot, H. 1997. Bacillariophyceae. Teil 2, Bacillariaceae, Epithemiaceae, Surirellaceae. In: Ettl, H., Gerloff, J., Heynig, H. \& Mollenhauer, D. (Eds), Süßwasserflora von Mitteleuropa, 2/2. Gustav Fischer, Stuttgart, 596 pp.

Krammer, K. \& Lange-Bertalot, H. 2000. Bacillariophyceae. Teil 3, Centrales, Fragilariaceae, Eunotiaceae. In: Ettl, H., Gerloff, J., Heynig, H. \& Mollenhauer, D. (Eds), Süßwasserflora von Mitteleuropa (2nd edn), 2/3. Gustav Fischer, Stuttgart, 599 pp.

Krasske, G. 1938. Beiträge zur Kenntnis der Diatomeen-Vegetation von Island und Spitzbergen. Archiv für Hydrobiologie, 33: 503-533.

Lagerstedt, N.G.W. 1873. Sötvattens-Diatomaceer fran Spetsbergen och Beeren Einland. Kungliga Vetenskaps Svenska Akademiens Handlingar, 1/14: 1-52.

Lange-Bertalot, H. 2001. Navicula sensu stricto 10 Genera Separated from Navicula sensu lato Frustulia. In: Lange-Bertalot, H. (Ed.), Diatoms of Europe, 2. A.R.G. Gantner, Ruggell, Germany, 1-526.

Lange-Bertalot, H. \& Genkal, S.I. 1999. Diatoms from Siberia I. Islands in the Arctic Ocean (Yugorsky Shar Strait). In: Lange-Bertalot, H (Ed.), Iconographia Diatomologica, 6. Koeltz Scientific Books, Koenigstein, 1-271.

Lange-Bertalot, H. \& Metzeltin, D. 1996. Ecology - Diversity -Taxonomy. Indicators of oligotrophy - 800 taxa representative of three ecologically distinct lake types. In: Lange-Bertalot, H. (Ed.), Iconographia Diatomologica, 2. Koeltz Scientific Books, Koenigstein, $1-390$.

Lange-Bertalot, H. \& Moser, G. 1994. Brachysira Monographie der Gattung. Bibliotheca Diatomologica, 29. J. Cramer, Stuttgart, 1-212.

Lyngbye, H.C. 1819. Tentamen hydrophytologiae Danicae continens omnia hydrophyta cryptogama Daniae, Holsatiae, Foeroeae, Islandiae, Groenlandiae hucusque cognita, systematicae disposita, descripta et iconibus illustrata, adjectis simul speciebus Norvegicis. Hafniae, Copenhagen.

Metzeltin, D. \& Lange-Bertalot, H. 1998. Tropical Diatoms of South America. In: Lange-Bertalot, H. (Ed.), Iconographia Diatomologica, 5. Koeltz Scientific Books, Koenigstein, 1-695.

Metzeltin, D. \& Witkowski, A. 1996. Diatomeen der Bären-Insel. In: Lange-Bertalot, H. (Ed.), Iconografia Diatomologica, 4. Koeltz Scientific Books, Koenigstein, 1-232.

Østrup, E. 1897. Kyst-Diatomeer fra Groenland. Meddelelser om Grönland, 15: 305-362.
Østrup, E. 1901. Freshwater diatoms from the Faeroes. Botany of the Faeroes, 1: 260-290.

Østrup, E. 1903. Diatoms from the marine algae of the Faeroes. In: Boergesen, F. (Ed.), The algae of the Faeroes. Botany of the Faeroes, 2: $533-557$

Podzorski, A.C. 1985. An Illustrated and Annotated Check-List of Diatoms from the Black River Waterways, St. Elizabeth, Jamaica. Bibliotheca Diatomologica, 7. J. Cramer, Stuttgart, 1-177.

Reichardt, E. 1999. Zur Revision der Gattung Gomphonema. In: Lange-Bertalot, H. (Ed.), Iconographia Diatomologica, 8. Koeltz Scientific Books, Koenigstein, 1-203.

Round, F.E., Crawford, R.M. \& Mann, D.G. 1990. The diatoms. Biology and morphology of the genera. Cambridge University Press, Cambridge, $747 \mathrm{pp}$.

Sala, S.E., Guerrero, J.M. \& Ferrario, M.E. 1993. Redefinition of Reimeria sinuata (Gregory) Kociolek \& Stoermer and recognition of Reimeria uniseriata nov. spec. Diatom Research, 8: 439-446.

Schrader, H. \& Gersonde, R. 1978. Diatoms and silicoflagellates. Utrecht Micropaleontological Bulletin, 17: 129-176.

Schrader, H., Isrenn, K., Swanberg, N., Paetzel, M. \& Saethre, T. 1993a. Early Holocene Diatom Pulse in the Norwegian Sea and its paleoceanographic significance. Diatom Research, 8: 117-130.

Schrader, H., Lindström Swanberg, I., Burckle, L.H. \& Grønlien, L. 1993 b. Diatoms in recent Atlantic $\left(20^{\circ} \mathrm{S}\right.$ to $70^{\circ} \mathrm{N}$ latitude) sediments: abundance patterns and what they mean. Hydrobiologia, 269/270: 129-135.

Stuiver, M. \& Reimer, P.J. 1993. Extended ${ }^{14} \mathrm{C}$ data-base and revised calib 3.0 C-14 age calibration program. Radiocarbon, 35: 215-230.

Stuiver, M., Reimer, P.J. \& Bard, E. 1998. et al. INTCAL98 Radiocarbon age calibration 24 000-0 cal bp. Radiocarbon, 40: 1041-1083.

Van Dam, H., Mertens, A. \& Sinkeldam, J. 1994. A coded checklist and ecological indicator values of freshwater diatoms from the Netherlands. Netherlands Journal of Aquatic Ecology, 28(1): 117-133.

van Weering, Tj.C.E., Nielsen, T., Kenyon, N.H., Akentieva, K. \& Kuijpers, A.H. 1998. Sediments and sedimentation at the NE Faeroe continental margin. contourites and large-scale sliding. Marine Geology, 152: 159-176.

Wachnicka, A. 1999. Changes in paleocirculation around Faeroe Islands in Holocene based on diatomological analyses. MSc Thesis. University of Szczecin, Poland.

Witak, M., Wachnicka, A., Kuijpers, A., Troelstra, S. \& Witkowski, A. in press. Holocene North Atlantic surface circulation and climate variability: Evidence from diatom records. The Holocene.

Witkowski, A., Lange-Bertalot, H. \& Metzeltin, D. 1996. The diatom species Fragilaria martyi (Héribaud) Lange-Bertalot, identity and ecology. Archiv Protist, 146: 281-292.

Witkowski, A., Lange-Bertalot, H. \& Metzeltin, D. 2000. Diatom Flora of Marine Coasts I. In: Lange-Bertalot, H. (Ed.), Iconographia Diatomologica, 7. A.R.G. Gantner, Ruggell, Germany, 1-905. 